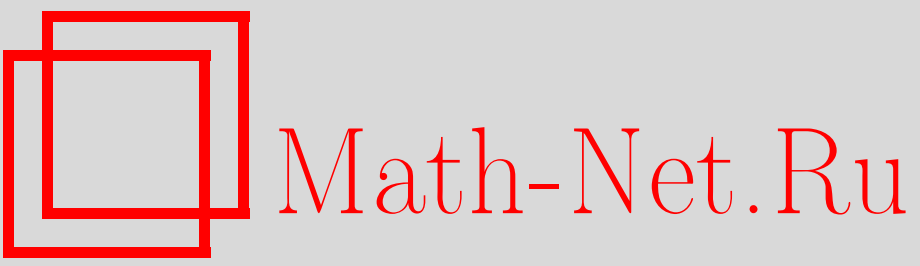

М. В. Козлов, О больших уклонениях максимума крамеровского случайного блуждания и процесса ожидания, Теория вероятн. и ее примен., 2013, том 58, выпуск 1, 81-116

DOI: https://doi.org/10.4213/tvp4495

Использование Общероссийского математического портала Math-Net.Ru подразумевает, что вы прочитали и согласны с пользовательским соглашением

http://www. mathnet.ru/rus/agreement

Параметры загрузки :

IP: 54.174 .149 .18

26 апреля 2023 г., 13:49:10 
2013

(c) 2013 г.

КОЗЛОВ М. В.*

\section{О БОЛЬШИХ УКЛОНЕНИЯХ МАКСИМУМА КРАМЕРОВСКОГО СЛУЧАЙНОГО БЛУЖДАНИЯ И ПРОЦЕССА ОЖИДАНИЯ}

В работе рассматриваются большие уклонения максимума $M_{n}$ отрезка случайного блуждания $S_{j}=\sum_{i=1}^{j} X_{i}, j \leqslant n$, и максимума $\mathscr{M}_{n}^{W}$ отрезка процесса ожидания $W_{j}$, заданного рекуррентной формулой $W_{j+1}:=\max \left(0, W_{j}+X_{j+1}\right)$, в предположении, что величины $X_{i}$ подчиняются правостороннему условию Крамера. Уточняются некоторые результаты А. А. Боровкова и Д. А. Коршунова об асимптотике вероятностей $\mathbf{P}\left(M_{n}>t n\right), \mathbf{E} X_{j}<0, n \rightarrow \infty$. Получены условные функциональные предельные теоремы для траектории случайного блуждания при условиях $T(t n)=k, T(x):=\inf \left(k: S_{k}>\right.$ $x), M_{n}>t n$ и процесса ожидания при условии $\mathscr{M}_{n}^{W}>t n$.

Ключевые слова и фразы: случайное блуждание, процесс ожидания, условие Крамера, большие уклонения максимума, условные предельные теоремы.

1. Введение. Пусть $X, X_{1}, X_{2}, \ldots$ - последовательность независимых одинаково распределенных невырожденных случайных величин (с.в.). Мы предполагаем, что математическое ожидание $\mathbf{E} X=: \mu_{0}$ конечно и что выполнено правостороннее условие Крамера

$$
\mathbf{E} e^{\lambda X}=: \theta(\lambda)<\infty \quad \text { при } 0<\lambda<\lambda_{+}, \lambda_{+}:=\sup (\lambda: \theta(\lambda)<\infty) \text {. }
$$

При значении $\mu_{0}<0$, если не оговорено противное, предполагаем, что

$$
\sup (\theta(\lambda): \lambda>0)>1, \quad \mathbf{P}(X>0)>0
$$

обозначим через $\varkappa$ положительный корень уравнения $\theta(\lambda)=1$.

Положим

$$
S_{n}:=\sum_{i=1}^{n} X_{i}, \quad S_{0}=0, \quad M_{n}:=\max \left(S_{j}: j \leqslant n\right), \quad M:=\sup \left(S_{j}: j \geqslant 0\right) .
$$

При $\mu_{0}<0$ вероятности больших уклонений $\mathbf{P}\left(M_{n}>x\right)$, $\mathbf{P}(M>x), n, x \rightarrow \infty$, подробно изучались в работах А. А. Боровкова и

*Московский государственный университет им. М. В. Ломоносова, механико-математический факультет, Москва, Россия; e-mail: kozlov_mikhail@mail.ru 
Д. А. Коршунова (см. [1], [2] и приведенную там библиографию). Случайная величина $M_{n}$ совпадает по распределению со с.в. $W_{n}$, определяемой рекуррентно [3]:

$$
W_{n+1}=\max \left(0, W_{n}+X_{n+1}\right), \quad W_{0}=0 .
$$

Исследование вероятностей $\mathbf{P}\left(W_{n}>x\right)$, основанное в работе [1] на формуле полной вероятности по значениям момента последнего возвращения процесса ожидания $W_{k}, k=0,1,2, \ldots, n-1$, в состояние 0 , приводит к соотношениям (см. [1, теорема 2$])$ :

$$
\mathbf{P}\left(M_{n}>x\right) \sim c e^{-\varkappa x}, \quad \mathbf{P}\left(M_{n}>x\right) \sim c\left(\frac{x}{n}\right) n^{-1 / 2} e^{-\psi(x / n) n}
$$

в зависимости от области изменения отношения $x / n, n, x \rightarrow \infty$; множители $c$ и $c(\cdot)$ определены в разделе $4, \psi(\cdot)$ - так называемая функция уклонений, определена в (1.9); символ $\sim$ соединяет выражения, отношение которых стремится к 1 .

Мы проводим прямой вероятностный анализ больших уклонений с.в. $M_{n}$, основанный на разложении

$$
\begin{gathered}
\mathbf{P}\left(M_{n}>t n\right)=\sum_{k=1}^{n} \mathbf{P}(T(t n)=k), \\
T(x):=\inf \left(k: S_{k}>x\right), \quad x>0, \quad t>\mu_{0}^{+},
\end{gathered}
$$

где $\mu_{0}^{+}$определено в (1.8).

Близкой по методу является работа А. В. Шкляева [4], где асимптотика вероятностей больших уклонений максимума выведена в случае $\mu_{0} \geqslant 0$.

Опираясь на классическую работу В. В. Петрова [5], в которой получена равномерная по $t$ асимптотика

$$
\mathbf{P}\left(S_{n}>t n\right) \sim c_{0}(t) n^{-1 / 2} e^{-\psi(t) n}, \quad n \rightarrow \infty, \quad t>\mu_{0},
$$

мы выводим соотношение (теорема 3.1)

$$
\mathbf{P}(T(t n)=n) \equiv \mathbf{P}\left(S_{n}>t n, M_{n-1} \leqslant t n\right) \sim c_{1}(t) n^{-1 / 2} e^{-\psi(t) n}, \quad n \rightarrow \infty,
$$

и применяем его к вероятностям

$$
\mathbf{P}(T(t n)=k)=\mathbf{P}\left(S_{k}>\frac{t n}{k} k, M_{k-1} \leqslant \frac{t n}{k} k\right) .
$$

В результате, в частности, получаются локальные предельные теоремы для вероятностей (1.6) в области больших уклонений максимума. Попутно заново выводятся теоремы о вероятностях больших уклонений $\mathbf{P}\left(M_{n}>t n\right)$ (теоремы 4.1, 4.2, 4.4), установленные ранее в [1] и [4]. 
При этом в случае $\mu_{0}<0$ удается несколько расширить область действия второй из асимптотик (1.2) (теорема 4.3). Полученные результаты применяются к нахождению асимптотики вероятностей больших уклонений максимума $\mathscr{M}_{n}^{W}:=\max \left(W_{j}: j \leqslant n\right)$ процесса ожидания (теоремы 5.3-5.5). Выводятся условные предельные теоремы для случайного блуждания и процесса ожидания в предположении большого уклонения максимума (разделы 6,7 ).

Введем обозначения:

$$
\begin{gathered}
\frac{d}{d \lambda} \ln \theta(\lambda)=: \mu(\lambda) \equiv \mu_{\lambda}, \quad \frac{d}{d \lambda} \mu(\lambda)=: \sigma^{2}(\lambda) \equiv \sigma_{\lambda}^{2}, \\
F_{\lambda}(x):=\theta^{-1}(\lambda) \int_{-\infty}^{x} e^{\lambda y} d F(y), \quad \lambda \in\left(0, \lambda_{+}\right), \\
\mu_{+}:=\lim _{\lambda \rightarrow \lambda_{+}} \mu(\lambda) \leqslant \infty, \quad \mu_{0}^{+}:=\max \left(0, \mu_{0}\right) .
\end{gathered}
$$

Соотношение (1.7) называют преобразованием Крамера функции распределения $F$. Среднее и дисперсия функции распределения $F_{\lambda}$ равны $\mu_{\lambda}, \sigma_{\lambda}^{2}$.

Функции $\theta(\lambda)$ и $\ln \theta(\lambda)$ строго выпуклы, функция $\mu(\lambda)$ строго возрастает, функция $\sigma(\lambda), \lambda \in\left(0, \lambda_{+}\right)$, положительна и непрерывна. Обратная к $\mu(\lambda)$ функция $\lambda(\mu) \equiv \lambda_{\mu}$ определена и возрастает на $\left[\mu_{0}, \mu_{+}\right), \lambda(\mu)>0$ при $\mu>\mu_{0}, \lambda\left(\mu_{0}\right)=0$. Множитель $c_{0}(t)$ в (1.4) равен в нерешетчатом случае $\left(\sqrt{2 \pi} \lambda_{t} \sigma\left(\lambda_{t}\right)\right)^{-1}$. Равномерность по $t$ в (1.4) имеет место на отрезках $[\alpha, \beta] \subseteq\left(\mu_{0}, \mu_{+}\right)$. Функция уклонений с.в. $X$ или функции распределения $F$

$$
\psi(t):=\sup _{\lambda>0}(t \lambda-\ln \theta(\lambda))=t \lambda_{t}-\ln \theta\left(\lambda_{t}\right), \quad t \in\left[\mu_{0}, \mu_{+}\right),
$$

возрастает и выпукла,

$$
\frac{d}{d t} \psi(t)=\lambda(t), \quad \frac{d^{2}}{d t^{2}} \psi(t)=\frac{d}{d t} \lambda(t)=\sigma^{-2}\left(\lambda_{t}\right)
$$

Для любого $t \in\left[\mu_{0}, \mu_{+}\right)$введем последовательность независимых одинаково распределенных с.в. $X^{(t)}, X_{1}^{(t)}, X_{2}^{(t)}, \ldots$ с функцией распределения $F_{\lambda(t)}$; при этом $\mathbf{E} X^{(t)}=t$, дисперсия $\mathbf{D} X^{(t)}=\sigma^{2}\left(\lambda_{t}\right)$ конечна для $t>\mu_{0}$,

$$
\mathbf{E} e^{\lambda X^{(t)}}=: \theta^{(t)}(\lambda)=\frac{\theta\left(\lambda_{t}+\lambda\right)}{\theta\left(\lambda_{t}\right)}<\infty, \quad \lambda \in\left[-\lambda_{t}, \lambda_{+}-\lambda_{t}\right) .
$$

Функция уклонений с.в. $X^{(t)}$ равна $\psi^{(t)}(s)=\psi(s)-\psi(t)-(s-t) \lambda_{t}$, $s \in\left[\mu_{0}, \mu_{+}\right)$. Функцию, представляющую касательную к функции $\psi(t)$ в точке $t=\mu_{\lambda}$, обозначим через $\psi(t ; \lambda)$ :

$$
\psi(t ; \lambda)=\psi\left(\mu_{\lambda}\right)+\lambda\left(t-\mu_{\lambda}\right) \equiv \lambda t-\ln \theta(\lambda) .
$$


Положим $L_{1 n}:=\min \left(S_{j}, 1 \leqslant j \leqslant n\right), S_{n}^{(t)}:=\sum_{i=1}^{n} X_{i}^{(t)}, S_{0}^{(t)}=0$, $L_{1 n}^{(t)}:=\min \left(S_{j}^{(t)}, 1 \leqslant j \leqslant n\right), L_{1}^{(t)}:=\inf \left(S_{j}^{(t)}, j \geqslant 1\right) ;$ будем считать, что $X^{\left(\mu_{0}\right)} \equiv X, S_{n}^{\left(\mu_{0}\right)} \equiv S_{n}, \psi^{\left(\mu_{0}\right)} \equiv \psi$ и т.п. Имеют место следующие неравенства для хвостов распределений (см., например, [6]):

$$
\begin{aligned}
& \mathbf{P}\left(S_{n}^{(t)}>s n\right) \leqslant e^{-\psi^{(t)}(s) n}, \quad s \in\left(t, \mu_{+}\right), \\
& \mathbf{P}\left(S_{n}^{(t)}<s n\right) \leqslant e^{-\psi^{(t)}(s) n}, \quad s \in\left(\mu_{0}, t\right), \\
& \mathbf{P}\left(L_{1 n}^{(t)} \leqslant-y\right) \leqslant e^{\bar{q}(t) y}, \quad \bar{q}(t):=\inf \left(\lambda: \theta^{(t)}(\lambda) \leqslant 1\right)<0, \quad t, y>0, \\
& \mathbf{P}\left(S_{n}>t n\right) \leqslant e^{-\psi\left(t, \lambda\left(t_{1}\right)\right) n} \quad \text { при } t \geqslant t_{1} \in\left(\mu_{0}, \mu_{+}\right) .
\end{aligned}
$$

Асимптотическое разложение до члена порядка $n^{-1 / 2}$ для функции распределения нормированных сумм $\left(S_{n}^{(t)}-t n\right) /\left(\sigma\left(\lambda_{t}\right) \sqrt{n}\right)$ выполняется равномерно по $t$ из любого отрезка, содержащегося в $\left(\mu_{0}, \mu_{+}\right)$(см. [1]), так что (см. [6]) при любом $\Delta>0$ равномерно по $y=o(\sqrt{n})$ и $t \in[\alpha, \beta] \subseteq$ $\left(\mu_{0}, \mu_{+}\right)$

$$
\mathbf{P}\left(S_{n}^{(t)}-t n \in[y, y+\Delta]\right) \sim \Delta\left(\sqrt{2 \pi n} \sigma\left(\lambda_{t}\right)\right)^{-1}, \quad n \rightarrow \infty .
$$

Ниже используются обозначения: $\varphi(\cdot)$ и $\Phi(\cdot)$ - соответственно плотность и функция распределения стандартного нормального закона, $w_{0}(s), 0 \leqslant s \leqslant 1,-$ броуновский мост $\left(w_{0}(s):=w(s)-s w(1)\right.$, где $w(s)-$ стандартное броуновское движение).

В формулировках теорем предполагается, что $\mu_{0}=\mathbf{E} X$ конечно, выполнено условие (A1), а при $\mu_{0}<0$ предполагается также, что $\mathbf{P}(X>0)>0$ и выполнено условие (А2), исключая теоремы 5.1, 6.1-6.5. Распределение вероятностей с.в. $X$ - невырожденное и нерешетчатое, решетчатый случай не вызывает затруднений. Ссылки в формулировках на работы [1], [4] указывают на приоритет.

Знаки эквивалентности $\sim$ и стремления $\longrightarrow, \stackrel{d}{\longrightarrow}$ в большинстве случаев будут относиться к условию $n \rightarrow \infty$, указание на это условие мы, как правило, будем опускать.

2. Фундаментальная лемма. Положим $l_{n}:=\left[n^{\rho}\right], 0<\rho<1$, где квадратные скобки означают целую часть заключенного в них числа. Предполагаем, что выполнено условие (A1), а распределение с.в. $X-$ нерешетчатое.

Лемма 2.1. (i) При $n \rightarrow \infty$ и любом $\Delta>0$ соотношение

$$
\mathbf{P}\left(S_{n}^{(t)}-t n \in[y, y+\Delta], L_{1 n}^{(t)} \geqslant x\right) \sim \Delta\left(\sqrt{2 \pi n} \sigma\left(\lambda_{t}\right)\right)^{-1} \mathbf{P}\left(L_{1 l_{n}}^{(t)} \geqslant x\right)
$$

выполняется равномерно по $t$ из любого отрезка $[\alpha, \beta] \subseteq\left(\mu_{0}^{+}, \mu_{+}\right)$, где $\mu_{0}^{+}=\max \left(0, \mu_{0}\right)$, равномерно по $y=o(\sqrt{n})$ и по $x \leqslant \delta l_{n}$ для $0<\delta<\alpha$.

(ii) Равномерно по $t$ и $x$, указанньм в $n$. (i),

$$
\mathbf{P}\left(L_{1 n}^{(t)} \geqslant x\right) \longrightarrow \mathbf{P}\left(L_{1}^{(t)} \geqslant x\right) ;
$$


в правой части (2.1) можно $L_{1 l_{n}}^{(t)}$ заменить на $L_{1}^{(t)}$; вероятность $\mathbf{P}\left(L_{1}^{(t)} \geqslant x\right)$ непрерьвна по $t$.

(iii) Равномерно по $t$, указанньлм в $n$. (i),

$$
\int_{0}^{\infty} e^{-\lambda_{t} x} \mathbf{P}\left(L_{1 n}^{(t)} \geqslant x\right) d x \longrightarrow \int_{0}^{\infty} e^{-\lambda_{t} x} \mathbf{P}\left(L_{1}^{(t)} \geqslant x\right) d x
$$

оба интеграла в (2.3) непрерьвны по $t$.

(iv) Для любого $\Delta>0$, любых $j u 0 \leqslant a_{i}<b_{i}, 1 \leqslant i \leqslant j$, равномерно по $t, y$ и $x$, указанныл в $n$. (i), выполняется соотношение

$$
\begin{aligned}
& P\left(S_{n}^{(t)}-t n \in[y, y+\Delta], L_{1 n}^{(t)} \geqslant x, S_{i}^{(t)} \in\left[x+a_{i}, x+b_{i}\right], 1 \leqslant i \leqslant j\right) \\
& \sim \Delta\left(\sqrt{2 \pi n} \sigma\left(\lambda_{t}\right)\right)^{-1} \int_{x+a_{j}}^{x+b_{j}} \mathbf{P}\left(L_{1}^{(t)} \geqslant x-x_{j}\right) \\
& \quad \times \mathbf{P}\left(S_{i}^{(t)} \in\left[x+a_{i}, x+b_{i}\right], i<j, S_{j}^{(t)} \in d x_{j}\right) .
\end{aligned}
$$

Д о к а з а т е л ь с т в о. (i) Запишем (2.1) в следующей интегральной форме:

$$
\begin{gathered}
\int_{-\infty}^{\infty} \mathbf{P}\left(S_{l_{n}}^{(t)}-t l_{n} \in d u, \sum_{i=1}^{n-l_{n}} X_{l_{n}+i}^{(t)}-t\left(n-l_{n}\right) \in[y-u, y-u+\Delta]\right. \\
\left.L_{1 l_{n}}^{(t)} \geqslant x, \min \left(\sum_{i=1}^{j} X_{l_{n}+i}^{(t)}: 1 \leqslant j \leqslant n-l_{n}\right) \geqslant-\left(t l_{n}+u\right)+x\right) .
\end{gathered}
$$

Зафиксируем $0<\varepsilon<\alpha$. Применяя неравенства (1.10), (1.11) для оценки вероятности $\mathbf{P}\left(\left|S_{l_{n}}^{(t)}-t l_{n}\right|>\varepsilon l_{n}\right)$, получаем, что она экспоненциально мала по $l_{n}$. В (2.5) сократим область интегрирования до отрезка $\left[-\varepsilon l_{n}, \varepsilon l_{n}\right]$, не нарушая объявленной асимптотики (2.1). Для полученного интеграла выпишем оценку сверху, отбросив под знаком вероятности в (2.5) последнее событие:

$$
\int_{-\varepsilon l_{n}}^{\varepsilon l_{n}} \mathbf{P}\left(S_{n-l_{n}}^{(t)}-t\left(n-l_{n}\right) \in[y-u, y-u+\Delta]\right) \mathbf{P}\left(S_{l_{n}}^{(t)}-t l_{n} \in d u, L_{1 l_{n}}^{(t)} \geqslant x\right) .
$$

K подынтегральной функции в (2.6) применим соотношение (1.14) и получим для интеграла (2.6) эквивалентное (равномерно по указанным $t$ и у) выражение

$$
\Delta\left(\sqrt{2 \pi n} \sigma\left(\lambda_{t}\right)\right)^{-1} \mathbf{P}\left(\left|S_{l_{n}}^{(t)}-t l_{n}\right| \leqslant \varepsilon l_{n}, L_{1 l_{n}}^{(t)} \geqslant x\right)
$$

Первое событие под знаком вероятности в (2.7) отбросим, не нарушая отмеченной эквивалентности, и получим верхнюю асимптотическую границу для оцениваемой вероятности в (2.1):

$$
\Delta\left(\sqrt{2 \pi n} \sigma\left(\lambda_{t}\right)\right)^{-1} \mathbf{P}\left(L_{1 l_{n}}^{(t)} \geqslant x\right) .
$$


Для получения нижней границы заменим последнее событие под знаком вероятности в $(2.5)$ на событие

$$
\min \left(\sum_{i=1}^{j} X_{l_{n}+i}^{(t)}: 1 \leqslant j \leqslant n-l_{n}\right) \geqslant-(t-\varepsilon) l_{n}+x
$$

которое получается заменой переменной $u$ на ее минимальное значение $u=-\varepsilon l_{n}$. Зафиксируем параметр $\delta$ из условия леммы, полагая $0<\delta<$ $\alpha-\varepsilon$. При $x<\delta l_{n}$ имеем $-(t-\varepsilon) l_{n}+x>-(\alpha-\varepsilon-\delta) l_{n}$, так что вероятность события (2.9) отличается от единицы на экспоненциально малую по $l_{n}$ величину. Отбрасывая это событие, приходим к интегралу (2.6) с уже найденной асимптотикой, которая дает нижнюю асимптотическую границу для вероятности в (2.1), совпадающую с верхней асимптотической границей (2.8).

(ii) Имеем:

$$
\begin{aligned}
0 & \leqslant \mathbf{P}\left(L_{1 n}^{(t)} \geqslant x\right)-\mathbf{P}\left(L_{1}^{(t)} \geqslant x\right)=\mathbf{P}\left(L_{1 n}^{(t)} \geqslant x, L_{1}^{(t)}<x\right) \\
& \leqslant \int_{-\varepsilon n}^{\varepsilon n} \mathbf{P}\left(S_{n}^{(t)}-t n \in d u, L_{1 n}^{(t)} \geqslant x, L_{1}^{(t)}<x\right)+\mathbf{P}\left(\left|S_{n}^{(t)}-t n\right|>\varepsilon n\right) .
\end{aligned}
$$

Интеграл в (2.10) оценим сверху вероятностью

$$
\mathbf{P}\left(L_{1}^{(t)}<-(t-\varepsilon) n+x\right) \leqslant \mathbf{P}\left(L_{1}^{(t)}<-(\alpha-\varepsilon-\delta) n\right) \quad \text { при }|x| \leqslant \delta n .
$$

При $0<\delta<\alpha-\varepsilon$ она экспоненциально мала по $n$. Второе слагаемое в правой части (2.10) экспоненциально мало по $n$ в силу неравенств (1.10), (1.11). Соотношение (2.2) установлено.

Легко проверить, что вероятность $\mathbf{P}\left(L_{1 n}^{(t)} \geqslant x\right)$ непрерывна по $t$. Из (2.2) следует, что вероятность $\mathbf{P}\left(L_{1}^{(t)} \geqslant x\right)$ также непрерывна по $t$.

(iii) В интегралах (2.3) сократим область интегрирования до отрезка $[0, \delta n]$ и, воспользовавшись соотношением (2.2), получим (2.3). Далее, положим $s_{j}:=\sum_{i=1}^{j} x_{i}$ и перепишем интеграл в левой части $(2.3)$ в виде

$$
\begin{aligned}
\int_{0}^{\infty} e^{-\lambda_{t} x} & {\left[\int_{\left\{s_{j} \geqslant x, j=1, \ldots, n\right\}} \cdots \int_{\left\{\left(x, x_{1}, \ldots, x_{n}\right): x \geqslant 0, s_{j} \geqslant x, j=1, \ldots, n\right\}} \theta\left(\lambda_{t}\right)^{-n} e^{\lambda_{t} s_{n}} \prod_{i=1}^{n} d F\left(x_{i}\right)\right] d x } \\
= & \theta^{n}\left(\lambda_{t}\right) \quad e^{\lambda_{t}\left(s_{n}-x\right)} d x \prod_{i=1}^{n} d F\left(x_{i}\right) .
\end{aligned}
$$

Он непрерывен по $t$, поскольку непрерывны функции $\lambda_{t}, \theta\left(\lambda_{t}\right)$, а подынтегральная функция равномерно интегрируема (в частности, равномерно по $t$ ). Непрерывность правого интеграла в (2.3) вытекает из равномерной сходимости (2.2). 
(iv) Вероятность в (2.4) представим в интегральной форме:

$$
\begin{array}{r}
\int_{a_{j}}^{b_{j}} \mathbf{P}\left(x_{j}+S_{n-j}^{(t)}-t n \in[y, y+\Delta], x_{j}+L_{1, n-j}^{(t)} \geqslant x\right) \\
\quad \times \mathbf{P}\left(S_{i}^{(t)} \in\left[x+a_{i}, x+b_{i}\right], i<j, S_{j}^{(t)} \in d x_{j}\right) .
\end{array}
$$

Ввиду п. (i) подынтегральная функция в (2.11) эквивалентна выражению $\Delta\left(\sqrt{2 \pi n} \sigma\left(\lambda_{t}\right)\right)^{-1} \mathbf{P}\left(L_{1}^{(t)} \geqslant x-x_{j}\right)$, что и приводит к соотношению (2.4).

3. Локальные предельные теоремы для момента достижения блужданием высокого уровня. Положим

$$
\begin{gathered}
a(t):=\int_{0}^{\infty} e^{-\lambda_{t} x} \mathbf{P}\left(L_{1}^{(t)} \geqslant x\right) d x, \quad c_{1}(t):=\lambda_{t} c_{0}(t) a(t), \\
\gamma:=\mu(\varkappa)>0(\varkappa>0-\text { корень уравнения } \theta(\lambda)=1), \lambda(\gamma)=\varkappa .
\end{gathered}
$$
$\left(\mu_{0}^{+}, \mu_{+}\right)$

Теорема 3.1. (i) Равномерно по $t$ из любого отрезка $[\alpha, \beta] \subseteq$

$$
\mathbf{P}(T(t n)=n) \equiv \mathbf{P}\left(S_{n}>t n, M_{n-1} \leqslant t n\right) \sim c_{1}(t) n^{-1 / 2} e^{-\psi(t) n} ;
$$

множитель $c_{1}(t)$, определенный в (3.1), непрерывен по $t$.

(ii) При $\mu_{0} \geqslant 0$ равномерно по $t \in[\alpha, \beta] \subseteq\left(\mu_{0}, \mu_{+}\right)$и по $k=o(\sqrt{n})$ выполняется соотношение

$$
\mathbf{P}(n-T(t n)=k) \sim \theta^{-k}\left(\lambda_{t}\right) c_{1}(t) n^{-1 / 2} e^{-\psi(t) n} .
$$

(iii) При $\mu_{0}<0$ соотношение (3.4) выполняется равномерно по $t \in$ $[\alpha, \beta] \subseteq\left(\gamma, \mu_{+}\right)$.

(iv) Равномерно по t, указанньлм в пn.(ii), (iii),

$$
\mathbf{P}\left(n-T(t n)=k \mid M_{n}>t n\right) \longrightarrow \theta^{-k}\left(\lambda_{t}\right)\left(1-\theta^{-1}\left(\lambda_{t}\right)\right), \quad k=0,1,2, \ldots
$$

Следствие 3.1. Равномерно по $t, k, n$ таким, ито $t n / k \in[\alpha, \beta] \subseteq$ $\left(\mu_{0}^{+}, \mu_{+}\right)$,

$$
\mathbf{P}(T(t n)=k) \sim c_{1}\left(\frac{t n}{k}\right) k^{-1 / 2} e^{-\psi(t n / k) k} .
$$

Следствие 3.2. Равномерно по $t$, указанным в теореме 3.1(i),

$$
\mathbf{P}\left(S_{n}-t n \leqslant y \mid T(t n)=n\right) \longrightarrow \frac{1}{a(t)} \int_{0}^{y} e^{-\lambda_{t} x} \mathbf{P}\left(L_{1}^{(t)} \geqslant x\right) d x, \quad y \geqslant 0 .
$$

Следствие 3.3. При $\mu_{0}<0$ равномерно по $t \in[\alpha, \gamma], \alpha>0, u$ $k-t \gamma^{-1} n=o\left(n^{2 / 3}\right)$

$$
\mathbf{P}(T(t n)=k) \sim \frac{b\left(t, t \gamma^{-1}\right)}{\sqrt{n}} \varphi\left(\frac{k-t \gamma^{-1} n}{b^{-1}\left(t, t \gamma^{-1}\right) \sqrt{n}}\right) \gamma^{-1} a(\gamma) e^{-\varkappa t n}
$$


где

$$
b\left(t, t \gamma^{-1}\right):=\sigma^{-1}(\varkappa) \gamma^{3 / 2} t^{-1 / 2}
$$

(cм. (3.22)).

3.1. Доказательство теоремы 3.1(i). Положим $s_{n}:=\sum_{i=1}^{n} x_{i}$, $m_{n}:=\max \left(s_{k}: 0 \leqslant k \leqslant n\right)$ и представим вероятность в $(3.3)$ в интегральной форме:

$$
\int \ldots \int_{\left\{m_{n-1} \leqslant t n, s_{n}>t n\right\}} \prod_{i=1}^{n} d F\left(x_{i}\right) .
$$

Сделав в (3.10) замену меры (1.7) при $\lambda=\lambda_{t}$, получим:

$$
\begin{aligned}
\theta^{n}\left(\lambda_{t}\right) & \int \ldots \int_{\left\{m_{n-1} \leqslant t n, s_{n}>t n\right\}} e^{-\lambda_{t} s_{n}} \prod_{i=1}^{n} d F_{\lambda_{t}}\left(x_{i}\right) \\
= & e^{-\psi(t) n} \int_{\left\{m_{n-1} \leqslant t n, s_{n}>t n\right\}} e^{-\lambda_{t}\left(s_{n}-t n\right)} \prod_{i=1}^{n} d F_{\lambda_{t}}\left(x_{i}\right) .
\end{aligned}
$$

Перепишем интеграл в (3.11) в виде

$$
\mathbf{E}\left(e^{-\lambda_{t}\left(S_{n}^{(t)}-t n\right)} ; S_{n}^{(t)}>t n, M_{n-1}^{(t)} \leqslant t n\right), \quad M_{n}^{(t)}:=\max \left(S_{j}^{(t)}: j \leqslant n\right) .
$$

От случайного блуждания $S_{k}^{(t)}, 1 \leqslant k \leqslant n$, перейдем к обращенному блужданию $\widehat{S}_{k}^{(t)}, 1 \leqslant k \leqslant n, \widehat{X}_{k}^{(t)}:=X_{n-k+1}^{(t)}, 1 \leqslant k \leqslant n$. Поскольку при этом $S_{n}^{(t)}=\widehat{S}_{n}^{(t)}, M_{n-1}^{(t)}=\widehat{S}_{n}^{(t)}-\widehat{L}_{1 n}^{(t)}$, то (3.12) переписывается в виде

$$
\begin{aligned}
& \mathbf{E}\left(e^{-\lambda_{t}\left(S_{n}^{(t)}-t n\right)} ; S_{n}^{(t)}>t n, L_{1 n}^{(t)} \geqslant S_{n}^{(t)}-t n\right) \\
& \quad=\int_{0+}^{\infty} e^{-\lambda_{t} x} \mathbf{P}\left(S_{n}^{(t)}-t n \in d x ; L_{1 n}^{(t)} \geqslant x\right) .
\end{aligned}
$$

Покажем, что интеграл (3.13) эквивалентен $c_{1}(t) n^{-1 / 2}$ при $n \rightarrow \infty$. Сократим в (3.13) область интегрирования до промежутка $\left(0, \delta \sqrt{l_{n}}\right)$, взяв $\delta>0$ из условия леммы 2.1, и оценим его суммами Дарбу-Стилтьеса по разбиению с шагом $\Delta>0$. Считая для простоты, что число $\delta \sqrt{l_{n}} / \Delta$ целое, выпишем эти суммы:

$$
\begin{aligned}
& \sum_{k<\delta \sqrt{l_{n}} / \Delta} e^{-\lambda_{t} k \Delta} \mathbf{P}\left(S_{n}^{(t)}-t n \in(k \Delta,(k+1) \Delta], L_{1 n}^{(t)} \geqslant k \Delta\right), \\
& \sum_{k<\delta \sqrt{l_{n}} / \Delta} e^{-\lambda_{t}(k+1) \Delta} \mathbf{P}\left(S_{n}^{(t)}-t n \in(k \Delta,(k+1) \Delta], L_{1 n}^{(t)} \geqslant(k+1) \Delta\right) .
\end{aligned}
$$

Применив лемму 2.1, получим для (3.14) эквивалентное (равномерно по указанным в теореме условиям) выражение:

$$
\left(\sqrt{2 \pi n} \sigma\left(\lambda_{t}\right)\right)^{-1} \sum_{0 \leqslant k<\delta \sqrt{l_{n}} / \Delta} e^{-\lambda_{t} k \Delta} \mathbf{P}\left(L_{1}^{(t)} \geqslant k \Delta\right) \Delta .
$$


Сумма в (3.16) является верхней суммой Дарбу для интеграла

$$
\int_{0}^{\delta \sqrt{l_{n}}} e^{-\lambda_{t} x} \mathbf{P}\left(L_{1}^{(t)} \geqslant x\right) d x
$$

При $n \rightarrow \infty$ он сходится к $a(t)$ (см. (3.1)). Выбирая $\Delta>0$ сколь угодно малым, приблизим сумму в (3.16) к интегралу (3.17) и тем самым получим для вероятности в (3.3) асимптотическую верхнюю границу, совпадающую с правой частью соотношения (3.3).

Аналогично, для суммы (3.15) получим выражение (3.16) с областью суммирования $1 \leqslant k \leqslant \delta \sqrt{l_{n}} / \Delta$. Поскольку суммы в $(3.16)$ и полученном выражении отличаются не более чем на $\Delta$, приходим к заключению (3.3). Непрерывность $a(t)$ установлена в лемме 2.1. Теорема 3.1(i) доказана.

Следствие 3.1 вытекает из представления (1.6). Чтобы получить следствие 3.2 , вероятность $\mathbf{P}\left(S_{n}>t n+y, M_{n-1} \leqslant t n\right)$ приводим к виду (cp. с (3.10)-(3.15)) $e^{-\psi(t) n} \mathbf{E}\left(e^{-\lambda_{t}\left(S_{n}^{(t)}-t n\right)} ; S_{n}^{(t)}>t n+y, M_{n-1}^{(t)} \leqslant t n\right)$ и далее повторяем проведенные выше выкладки. Следствие 3.3 выводится в п. 3.3 .

3.2. Доказательство теоремы 3.1(ii)-(iv). Введем вспомогательную функцию

$$
g(t, u):=\psi\left(\frac{t}{u}\right) u, \quad t, u>0, \quad \frac{t}{u} \in\left(\mu_{0}^{+}, \mu_{+}\right),
$$

и выразим через нее показатель экспоненты в (3.6):

$$
\psi\left(\frac{t n}{k}\right) k=g\left(t, \frac{k}{n}\right) n
$$

Исследуем функцию $g(t, u)$ по переменной $u$. Дифференцируя, получаем:

$$
\begin{aligned}
\frac{\partial g(t, u)}{\partial u} & =-\lambda\left(\frac{t}{u}\right) \frac{t}{u}+\psi\left(\frac{t}{u}\right)=-\ln \theta\left(\lambda\left(\frac{t}{u}\right)\right) \\
\frac{\partial^{2} g(t, u)}{\partial u^{2}} & =\mu\left(\lambda\left(\frac{t}{u}\right)\right) \sigma^{-2}\left(\lambda\left(\frac{t}{u}\right)\right) \frac{t}{u^{2}}=\sigma^{-2}\left(\lambda\left(\frac{t}{u}\right)\right) \frac{t^{2}}{u^{3}} .
\end{aligned}
$$

Положим для краткости

$$
b^{2}(t, u):=\sigma^{-2}\left(\lambda\left(\frac{t}{u}\right)\right) \frac{t^{2}}{u^{3}} .
$$

Как видно, функция $g(t, u)$ выпукла по $u$, возрастает в области, где $\theta(\lambda(t / u))<1$, и убывает при $\theta(\lambda(t / u))>1$.

(ii) Воспользуемся соотношением (3.6) при $n-k=o(\sqrt{n})$. Пусть $\mu_{0} \geqslant 0$. Тогда $\theta(\lambda)>1$ при всех $\lambda \in\left(0, \lambda_{+}\right)$. Выражение (3.19) возрастает 
при уменьшении $k: k=n, n-1, n-2, \ldots$. Функцию $g(t, u)$ разложим по формуле Тейлора в точке $u=1$ (что соответствует $k=n$ ):

$$
g(t, u)=\psi(t)-\ln \theta\left(\lambda_{t}\right) \cdot(u-1)+\frac{1}{2} b^{2}\left(t, u^{\prime}\right)(u-1)^{2},
$$

где $u^{\prime}$ - точка между $u$ и 1 . Подставляя в $(3.23) u=k / n$, получаем

$$
\exp \left\{-g\left(t, \frac{k}{n}\right) n\right\}=e^{-\psi(t) n} \theta^{k-n}\left(\lambda_{t}\right)(1+o(1)),
$$

где член $o(1)$ равномерно мал по $t$ и по $k$ таким, что $n-k=o(\sqrt{n})$. Подставляя (3.24) в (3.6) и заменяя $k$ на $n-k$, получим соотношение (3.4).

(iii) При $\mu_{0}<0, t \in[\alpha, \beta] \subseteq\left(\gamma, \mu_{+}\right)$имеем $\theta(\lambda(t))>1$, и все предшествующие рассуждения проходят практически без изменений.

(iv) Соотношение (3.5) устанавливается в п. 4.1(iii).

3.3. Вывод следствия 3.3. В указанных предположениях функция $g(t, u)$, определенная в $(3.18)$, достигает минимума по переменной $u$ в точке $u=u_{t}$, удовлетворяющей уравнению $\theta(\lambda(t / u))=1$. В стороны от точки $u_{t}$ функция $g(t, u)$ возрастает. Из $(3.2)$ получаем: $\lambda\left(t / u_{t}\right)=\varkappa$, $t / u_{t}=\gamma, u_{t}=t / \gamma$. Разложение Тейлора функции $g(t, u)$ в точке $u_{t} \mathrm{c}$ учетом равенств (см. (3.21)-(3.23))

$$
\begin{gathered}
\left.\frac{\partial g(t, u)}{\partial u}\right|_{u=u_{t}}=-\ln \theta(\lambda(\gamma))=0,\left.\quad \frac{\partial^{2} g(t, u)}{\partial u^{2}}\right|_{u=u_{t}}=b^{2}\left(t, t \gamma^{-1}\right), \\
g\left(t, u_{t}\right)=\psi\left(\frac{t}{u_{t}}\right) u_{t}=\psi(\gamma) \frac{t}{\gamma}=(\gamma \varkappa-\ln \theta(\lambda(\gamma))) \frac{t}{\gamma}=\varkappa t
\end{gathered}
$$

принимает вид

$$
g(t, u)=\varkappa t+\frac{1}{2} b^{2}\left(t, t \gamma^{-1}\right)\left(u-t \gamma^{-1}\right)^{2}+O(1)\left(u-t \gamma^{-1}\right)^{3},
$$

где член $O(1)$ равномерно ограничен по $t \in[\alpha, \beta] \subseteq\left(0, \mu_{+}\right)$. Подставив в (3.26) $u=k / n$ при $k-t \gamma^{-1} n=o\left(n^{2 / 3}\right)$, а результат подстановки - в (3.6), приходим к (3.8).

\section{4. Асимптотика вероятностей больших уклонений макси- мума отрезка блуждания.} $\left(\mu_{0}, \mu_{+}\right)$

Теорема 4.1 (см. [4]). При $\mu_{0} \geqslant 0$ равномерно по $t \in[\alpha, \beta] \subseteq$

$$
\mathbf{P}\left(M_{n}>t n\right) \sim\left(1-\theta^{-1}\left(\lambda_{t}\right)\right)^{-1} c_{1}(t) n^{-1 / 2} e^{-\psi(t) n},
$$

где $c_{1}(t)$ определено в (3.1).

3 а м е ч а н и е. В [4] множитель при $n^{-1 / 2} e^{-\psi(t) n}$ в (4.1) имеет вид $c_{0}(t) b(t) \mathbf{P}\left(L_{1}^{(t)}>0\right)$, где

$$
b(t):=\sum_{k=1}^{\infty} \theta^{-k}\left(\lambda_{t}\right) \mathbf{P}\left(L_{1 k}<0\right), \quad c_{0}(t):=\left(\sqrt{2 \pi} \lambda_{t} \sigma\left(\lambda_{t}\right)\right)^{-1} .
$$


Теорема 4.2 (см. [1]). При $\mu_{0}<0$ равномерно по $t \in[\alpha, \beta] \subseteq$ $\left(\gamma, \mu_{+}\right)$выполняется соотношение (4.1).

3 а м е ч а н и е. Множитель при $n^{-1 / 2} e^{-\psi(t) n}$ в теореме 4.2 приведен в работе [1] в виде

$$
c_{0}(t)\left(1-\theta^{-1}\left(\lambda_{t}\right)\right)^{-1} d(t), \quad d(t):=\int_{0+}^{\infty} e^{\lambda_{t} x} \mathbf{P}\left(L_{1}^{(t)} \geqslant-x\right) d F(x) .
$$

Методы работы [4] действуют и в условиях теоремы 4.2.

Теорема 4.3. При $\mu_{0}<0$ соотношение (4.1) выполняется равномерно по $t \in\left[\gamma+n^{-1 / 2+\delta}, \beta\right], \beta<\mu_{+}$, значение $\delta>0$ можно взять сколь угодно мальм, а также можно положить $\delta=\delta_{n}$, где $\delta_{n} \longrightarrow 0$ так, uто $\delta_{n} \ln n \longrightarrow \infty$.

Теорема 4.4 (см. [1]). Пусть $\mu_{0}<0$ Тогда:

(i) равномерно по $t \in[\alpha, \beta] \subseteq(0, \gamma)$

$$
\mathbf{P}\left(M_{n}>t n\right) \sim \gamma^{-1} a(\gamma) e^{-\varkappa t n} ;
$$

(ii) после добавления в правую часть (4.3) множителя

$$
\Phi\left(\sqrt{n} b\left(t, t \gamma^{-1}\right)\left(1-t \gamma^{-1}\right)\right)
$$

соотношение (4.3) выполняется равномерно по $t \in[\alpha, \gamma], \alpha>0$;

(iii) соотношение (4.3) с множителем (4.4) в правой части выполняется равномерно по $t \in\left[\alpha, \gamma+n^{-1 / 3-\delta}\right]$, где $\delta>0$ можно взять сколь угодно мальм, а также положить $\delta=\delta_{n}$, где $\delta_{n} \longrightarrow 0$ так, что $\delta_{n} \geqslant A(\ln \ln n)(\ln n)^{-1}$ для подходящего $A$, зависящего от $\varkappa, \gamma$.

3 а м е ч а н и е. В работе [1] множитель при $e^{-\varkappa t n}$ в (4.3) получен в виде $(\varkappa \gamma)^{-1} d(\gamma)$, где $d(t)$ определено в $(4.2)$.

Из соотношений (4.3), (3.8) получаем: при $\mu_{0}<0$ равномерно по $t \in\left[\alpha, \beta_{n}\right], \alpha>0,\left(\gamma-\beta_{n}\right) \sqrt{n} \longrightarrow \infty$ u no k maкuм, umo $k-t \gamma^{-1} n=o\left(n^{2 / 3}\right)$,

$$
\mathbf{P}\left(T(t n)=k \mid M_{n}>t n\right) \sim \frac{b\left(t, t \gamma^{-1}\right)}{\sqrt{n}} \varphi\left(\frac{k-t \gamma^{-1} n}{b^{-1}\left(t, t \gamma^{-1}\right) \sqrt{n}}\right) .
$$

Положим

$$
\Psi(s, n):=\frac{1}{\sqrt{2 \pi n}} \sum_{l \geqslant 0} e^{-l^{2} /(2 n)-l s}=e^{s^{2} n / 2} \sum_{l \geqslant 0} \frac{1}{\sqrt{n}} \varphi\left(\frac{l+s n}{\sqrt{n}}\right) .
$$

Следующие утверждения очевидны: $n p u s \geqslant 0$ u натуральных $n$

$$
0 \leqslant \sum_{l \geqslant 0} \frac{1}{\sqrt{n}} \varphi\left(\frac{l+s n}{\sqrt{n}}\right)-(1-\Phi(s \sqrt{n})) \leqslant \frac{1}{\sqrt{2 \pi n}} e^{-s^{2} n / 2},
$$


$n р и s \sqrt{n} \geqslant 1$ нуль в левой части (4.7) можно заменить на вьражение в правой части с дополнительным множителем 1/2;

$$
\begin{aligned}
& \Psi(s, n) \sim \frac{1}{\sqrt{2 \pi n}}\left(1-e^{-s}\right)^{-1}, \quad \text { если } s \sqrt{n} \rightarrow \infty \\
& \Psi(s, n) \sim(1-\Phi(s \sqrt{n})) e^{s^{2} n / 2}, \quad \text { если } s \sqrt{n}=O(1) .
\end{aligned}
$$

Теорема 4.5. При $\mu_{0}<0$ равномерно по $t \in[\gamma, \beta], \beta<\mu_{+}$,

$$
\begin{aligned}
\mathbf{P}\left(M_{n}>t n\right) & \sim \Psi\left(\ln \theta\left(\lambda_{t}\right), b^{-2}(t, 1) n\right) b^{-1}(t, 1) \sqrt{2 \pi} c_{1}(t) e^{-\psi(t) n} \\
& =\Psi\left(\ln \theta\left(\lambda_{t}\right), \sigma^{2}\left(\lambda_{t}\right) t^{-2} n\right) t^{-1} a(t) e^{-\psi(t) n}
\end{aligned}
$$

3 а м е ч а н и е. Теоремы 4.3 и $4.4($ iii $)$ в диапазоне $t \in(\gamma+$ $\left.n^{-1 / 2+\delta^{\prime}}, \gamma+n^{-1 / 3-\delta^{\prime \prime}}\right)$ дают разные по форме асимптотики для вероятностей больших уклонений $\mathbf{P}\left(M_{n}>t n\right)$. Теорема 4.5 дает формально единое выражение для вероятностей больших уклонений при $t \in[\gamma, \beta]$, $\beta<\mu_{+}$. При $(t-\gamma) \sqrt{n} \rightarrow \infty$ оно эквивалентно правой части (4.1) (и получается подстановкой в $(4.8) s=\ln \theta\left(\lambda_{t}\right)$ и заменой $n$ на $\left.b^{-2}(t, 1) n\right)$. При $0 \leqslant(t-\gamma) \sqrt{n}=O(1)$ утверждение (4.8) совпадает с теоремой 4.4(iii).

Подведем итог: при $\mu_{0}<0$ имеет место эквивалентность

$$
\mathbf{P}\left(M_{n}>t n\right) \sim \begin{cases}\Phi\left(\sqrt{n} b\left(t, t \gamma^{-1}\right)\left(1-t \gamma^{-1}\right)\right) \gamma^{-1} a(\gamma) e^{-\varkappa t n} & \text { npu } t \in(0, \gamma], \\ \Psi\left(\ln \theta\left(\lambda_{t}\right), \sigma^{2}\left(\lambda_{t}\right) t^{-2} n\right) t^{-1} a(t) e^{-\psi(t) n} & \text { npu } t \in\left[\gamma, \mu_{+}\right),\end{cases}
$$

равномерная по $t \in[\alpha, \beta] \subseteq\left(0, \mu_{+}\right) ; \psi(\gamma)=\varkappa \gamma$.

4.1. Доказательство теорем 4.1, 4.2. (i) При $\mu_{0} \geqslant 0$ сумму в (1.3) разложим на части: (I) $k \geqslant n-\sqrt[3]{n}$, (II) $(1-\varepsilon) n<k<n-\sqrt[3]{n}$, (III) $k \leqslant(1-\varepsilon) n$, где $\varepsilon-$ любое фиксированное число из интервала $\left(0,1-\beta \mu_{+}^{-1}\right)$. Суммируя соотношение (3.4) по области (I), получаем выражение, эквивалентное правой части (4.1). Следовательно, остается проверить, что суммы по областям (II), (III) пренебрежимо малы.

Для слагаемых суммы по области (II) выполняется соотношение (3.6), так что эта сумма эквивалентна следующей (см. (3.19)):

$$
\sum_{(1-\varepsilon) n<k<n-\sqrt[3]{n}} c_{1}\left(\frac{t n}{k}\right) k^{-1 / 2} e^{-g(t, k / n) n}
$$

Функция $g(t, u)$ в рассматриваемом случае убывает по $u$ (см. (3.20)), так что в области (II), применяя разложение Тейлора (3.23), получаем

$$
\begin{aligned}
g\left(t, \frac{k}{n}\right) & >g\left(t, \frac{n-\sqrt[3]{n}}{n}\right)=g\left(t, 1-n^{-2 / 3}\right) \\
& =\psi(t)+\ln \theta\left(\lambda_{t}\right) \cdot n^{-2 / 3}+O(1) n^{-4 / 3} \\
e^{-g(t, k / n) n} & \leqslant e^{-\psi(t) n}\left(\theta\left(\lambda_{t}\right)+o(1)\right)^{-n^{1 / 3}} .
\end{aligned}
$$


Подставив (4.12) в (4.11), находим, что сумма по области (II) относительно мала.

Слагаемые суммы по области (III) оценим сверху вероятностями

$$
\mathbf{P}\left(S_{k}>t n\right)=\mathbf{P}\left(S_{k}>\frac{t n}{k} k\right)
$$

для которых, применяя оценку (1.13), получаем границу сверху:

$$
\exp \left\{-\psi\left(\frac{t n}{k}, \lambda\left(t_{1}\right)\right) k\right\}=\exp \left\{-\left\{\psi\left(\frac{t n}{k}, \lambda\left(t_{1}\right)\right) \frac{k}{n}\right\} n\right\},
$$

где значение $t_{1} \in\left(t, \mu_{+}\right)$можно выбрать любым, не превосходящим $t n / k$. Мы положим $t_{1}=t(1-\varepsilon / 2) /(1-\varepsilon)$, так что в области (III)

$$
t<t_{1}<\frac{t}{1-\varepsilon}<\frac{t n}{k} .
$$

Функция $\psi(v, \lambda)$ задает касательную в точке $v=\mu_{\lambda}$ к графику выпуклой и возрастающей функции $\psi(v), \psi(0)=0$, поэтому

$$
\frac{\psi(v, \lambda)}{v}>\frac{\psi\left(v_{1}, \lambda\right)}{v_{1}} \quad \text { при } v>v_{1}>0 .
$$

Подставляя в (4.16) $v=t n / k, v_{1}=t_{1}, \lambda=\lambda\left(t_{1}\right)$, получим

$$
\psi\left(\frac{t n}{k}, \lambda\left(t_{1}\right)\right) / \frac{t n}{k}>\frac{\psi\left(t_{1}\right)}{t_{1}}, \quad \text { или } \quad \psi\left(\frac{t n}{k}, \lambda\left(t_{1}\right)\right) \frac{k}{n}>t \frac{\psi\left(t_{1}\right)}{t_{1}} .
$$

Следовательно, выражение (4.14) не превосходит

$$
\exp \left\{-t \frac{\psi\left(t_{1}\right)}{t_{1}} n\right\}
$$

При $t<t_{1}$ выражение

$$
t \frac{\psi\left(t_{1}\right)}{t_{1}}-\psi(t)=t\left(\frac{\psi\left(t_{1}\right)}{t_{1}}-\frac{\psi(t)}{t}\right)
$$

положительно, так как функция $\psi(t)$ возрастает и выпукла. При $t_{1}=$ $t(1-\varepsilon / 2) /(1-\varepsilon)$ функция (4.19) непрерывна и положительна на отрезке $[\alpha, \beta]$, а потому принимает на нем положительное минимальное значение $\delta$. Таким образом, при всех $t \in[\alpha, \beta]$ выражение (4.18), а вместе с ним и (4.14), не превосходит $\exp \{-(\psi(t)+\delta) n\}$, т.е. сумма по области (III) пренебрежимо мала.

(ii) Рассуждения п. (i) без изменений переносятся на теорему 4.2.

(iii) Поделив обе части соотношения (3.4) на соответствующие части соотношения (4.1), приходим к (3.5). 
4.2. Доказательство теоремы 4.3. При $t \in\left[\gamma+n^{-1 / 2+\delta}, \beta\right]$ разложим сумму в (1.3) на части по следующим областям:

(I) $k \geqslant n-n^{(1-\delta) / 2}, \quad$ (II) $(1-\varepsilon) n<k<n-n^{(1-\delta) / 2}, \quad$ (III) $k \leqslant(1-\varepsilon) n$,

где $\varepsilon>0$ то же, что и в п. 4.1. Все три суммы оцениваются по схеме раздела 4.1. Суммируя соотношение (3.6) по области (I), получаем для суммы по этой области эквивалентное при $n \rightarrow \infty$ выражение

$$
\sum_{k \geqslant n-n^{(1-\delta) / 2}} c_{1}(t) n^{-1 / 2} e^{-g(t, k / n) n} .
$$

Из разложения (3.23) имеем равномерно по $k$ :

$$
\exp \left\{-g\left(t, \frac{k}{n}\right) n\right\}=e^{-\psi(t) n} \theta^{n-k}\left(\lambda_{t}\right)\left(1+O\left(n^{-\delta}\right)\right) .
$$

Суммируя $\theta^{n-k}\left(\lambda_{t}\right)$ по $k$ из области $(\mathrm{I})$, получаем величину

$$
\left(1-\theta^{-1}\left(\lambda_{t}\right)\right)^{-1}\left(1-\left(\theta\left(\lambda_{t}\right)\right)^{-\left[n^{(1-\delta) / 2}\right]-1}\right) .
$$

Второй множитель в (4.22) стремится к 1 , так как

$$
\begin{gathered}
\ln \theta\left(\lambda_{t}\right) \geqslant \ln \theta\left(\lambda\left(\gamma+n^{-1 / 2+\delta}\right)\right)=\left(\sigma^{-2}(\varkappa) \gamma+o(1)\right) n^{-1 / 2+\delta} \\
-n^{(1-\delta) / 2} \ln \theta\left(\lambda_{t}\right) \leqslant-\left(\sigma^{-2}(\varkappa) \gamma+o(1)\right) n^{\delta / 2} \longrightarrow-\infty .
\end{gathered}
$$

Таким образом, сумма (4.20) эквивалентна правой части (4.1).

Учитывая, что по переменной $u$ функция $g(t, u)$ выпуклая и убывающая, в области (II) имеем (см. (4.23)):

$$
\begin{aligned}
& g\left(t, \frac{k}{n}\right)>g\left(t, 1-n^{-1 / 2-\delta / 2}\right)>\psi(t)+n^{-1 / 2-\delta / 2} \ln \theta\left(\lambda_{t}\right) \\
& \geqslant \psi(t)+n^{-1 / 2-\delta / 2} \ln \theta\left(\lambda\left(\gamma+n^{-1 / 2+\delta}\right)\right)=\psi(t)+\left(\sigma^{-2}(\varkappa) \gamma+o(1)\right) n^{-1+\delta / 2}, \\
& \exp \left\{-g\left(t, \frac{k}{n}\right) n\right\} \leqslant e^{-\psi(t) n} \exp \left\{\left(\sigma^{-2}(\varkappa) \gamma+o(1)\right) n^{\delta / 2}\right\} .
\end{aligned}
$$

Тем самым, сумма по области (II) пренебрежимо мала. Оценивание суммы по области (III) проходит без изменений.

Просматривая проведенные выкладки, убеждаемся в сохранении выводов, если $\delta_{n} \longrightarrow 0$ таким образом, что $n^{\delta_{n}} \rightarrow \infty$.

4.3. Доказательство теоремы 4.4. (i) При $t \in[\alpha, \beta] \subseteq(0, \gamma)$ сумму в (1.3) разложим на части:

$$
\text { (I) }\left|k-t \gamma^{-1} n\right| \leqslant n^{5 / 8}, \quad \text { (II) } t \gamma^{-1} n+n^{5 / 8}<k \leqslant n,
$$

(III) $t(\gamma+\varepsilon)^{-1} n \leqslant k<t \gamma^{-1} n-n^{5 / 8}, \quad$ (IV) $k<t(\gamma+\varepsilon)^{-1} n$, 
где $\varepsilon>0$ выберем из условия $\gamma+\varepsilon<\mu_{+}$. Просуммируем соотношение (3.8) по области (I) и представим результат в виде произведения следующих двух выражений:

$$
\begin{gathered}
\frac{b\left(t, t \gamma^{-1}\right)}{\sqrt{2 \pi n}} \sum_{\left|k-t \gamma^{-1} n\right|<n^{5 / 8}} \exp \left\{-\frac{1}{2} \frac{\left(k-t \gamma^{-1} n\right)^{2}}{b^{-2}\left(t, t \gamma^{-1}\right) n}\right\}, \\
\sqrt{2 \pi} b^{-1}\left(t, t \gamma^{-1}\right) t^{-1 / 2} \gamma^{1 / 2} c_{1}(\gamma) e^{-\varkappa t n} \equiv \gamma^{-1} a(\gamma) e^{-\varkappa t n} .
\end{gathered}
$$

Сомножитель (4.24) стремится при $n \rightarrow \infty$ к интегралу от нормальной плотности по всей прямой, так что произведение (4.24) и (4.25) эквивалентно выражению (4.25), которое совпадает с правой частью (4.3).

В областях (II), (III) имеем: $\alpha \leqslant t \leqslant t n / k \leqslant \gamma+\varepsilon<\mu_{+}$, так что к соответствующим слагаемым применима асимптотика (3.6). Поэтому сумма по области (II) эквивалентна при $n \rightarrow \infty$ следующей (см. (3.19)):

$$
\sum_{t \gamma^{-1} n+n^{5 / 8} \leqslant k \leqslant n} c_{1}\left(\frac{t n}{k}\right) k^{-1 / 2} e^{-g(t, k / n) n} .
$$

Функция $g(t, u)$ возрастает по $u$ на $\left(t \gamma^{-1}, 1\right)$, так что (см. $\left.(3.26)\right)$

$$
\begin{gathered}
g\left(t, \frac{k}{n}\right) \geqslant g\left(t, t \gamma^{-1}+n^{-3 / 8}\right)=\varkappa t+\frac{1}{2} b^{2}\left(t, t \gamma^{-1}\right) n^{-6 / 8}+O(1) n^{-9 / 8}, \\
\exp \left\{-g\left(t, \frac{k}{n}\right) n\right\} \leqslant e^{-\varkappa t n} \exp \left\{-\frac{1}{2} b\left(t, t \gamma^{-1}\right) n^{1 / 4}+O(1) n^{-1 / 8}\right\}
\end{gathered}
$$

в области (II). Подставив (4.27) в (4.26), убеждаемся, что сумма (II) пренебрежимо мала. Совершенно аналогично оценивается сумма (III).

Сумму (IV) оценим подобно сумме (III) в доказательстве теоремы 4.1. Именно, используем оценку (4.14) для вероятностей (4.13), в которой положим $t_{1}=\gamma+\varepsilon$. Поскольку (cp. с (4.16))

$$
\frac{\psi(v, \lambda(\gamma+\varepsilon))}{\lambda(\gamma+\varepsilon)}>\frac{\psi(\gamma+\varepsilon, \lambda(\gamma+\varepsilon))}{\lambda(\gamma+\varepsilon)} \equiv \frac{\psi(\gamma+\varepsilon)}{\gamma+\varepsilon} \quad \text { при } v>\gamma+\varepsilon,
$$

то при $t n / k>\gamma+\varepsilon\left(\right.$ cp. с (4.17)) $\psi\left(t n / k, \lambda\left(t_{1}\right)\right) k / n>t \psi\left(t_{1}\right) / t_{1}, t_{1}=\gamma+\varepsilon$. Так как (ср. с (4.19)) $t \psi\left(t_{1}\right) / t_{1}-\varkappa t=t\left(\psi\left(t_{1}\right) / t_{1}-\psi(\gamma) / \gamma\right)=: t \delta, \delta>0$, то слагаемые суммы (IV) не превосходят (см. (4.18)) $\exp \left\{-t\left(\psi\left(t_{1}\right) / t_{1}\right) n\right\}=$ $\exp \{-(\varkappa+\delta) n\}$. Тем самым, сумма (IV) пренебрежимо мала, и первая часть теоремы доказана.

(ii) При $t \in[\alpha, \gamma]$ оценивание сумм (III), (IV) и суммы (II) (когда она не пуста, т.е. $\left.t \gamma^{-1} n+n^{5 / 8}<n\right)$ проходит без изменений. Подмножество (I) запишем в следующем виде: (I) $t \gamma^{-1} n-n^{5 / 8} \leqslant k \leqslant$ $\min \left(t \gamma^{-1} n+n^{5 / 8}, n\right)$. Сумма (4.24) по области (I) эквивалента при $n \rightarrow \infty$ интегралу от стандартной нормальной плотности по области 
$\left(-\infty, \sqrt{n} b\left(t, t \gamma^{-1}\right)\left(1-t \gamma^{-1}\right)\right)$, что привносит в правую часть соотношения (4.3) множитель (4.4).

(iii) При $t \in\left[\gamma, \gamma+n^{-1 / 3-\delta}\right]$ сумма в (1.3) разбивается на части (I), (III), (IV), указанные выше (часть (II) - пустая), при этом область (I) запишем в виде (I) $t \gamma^{-1} n-n^{1 / 2+\delta} \leqslant k \leqslant n$. Оценивание частей (III), (IV) проходит без изменений. Для оценивания суммы (I) разложим $g(t, u)$ как функцию двух переменных по формуле Тейлора в точке $t=\gamma, u=1$ до членов второго порядка:

$$
\begin{aligned}
\varkappa \gamma & +\varkappa(t-\gamma)+\frac{1}{2} \sigma^{-2}(\varkappa)(t-\gamma)^{2}-\sigma^{-2}(\varkappa) \gamma(t-\gamma)(u-1) \\
& +\frac{1}{2} \sigma^{-2}(\varkappa) \gamma^{2}(u-1)^{2} \\
= & \varkappa \gamma+\frac{1}{2} \sigma^{-2}(\varkappa)((t-\gamma)-\gamma(u-1))^{2}=\varkappa \gamma+\frac{1}{2} \sigma^{-2}(\varkappa) \gamma^{2}\left(u-t \gamma^{-1}\right)^{2} .
\end{aligned}
$$

Остаточный член при $t \rightarrow \gamma, u \rightarrow 1$ не превосходит по порядку максимума из выражений

$$
(t-\gamma)^{2}(u-1), \quad(u-1)^{2}(t-\gamma), \quad(t-\gamma)^{3}, \quad(u-1)^{3} .
$$

При рассматриваемых $t$ и $k$ имеем: $t-\gamma \leqslant n^{-1 / 3-\delta}, 1-k / n \leqslant n^{-1 / 2+\delta}$. Выражения (4.28) не превосходят соответственно $n^{-7 / 6-\delta}, n^{-4 / 3+\delta}, n^{-1-3 \delta}$, $n^{-3 / 2+3 \delta}$. Отсюда следует, что в области (I) (см. (3.8))

$$
\mathbf{P}(T(t n)=k) \sim c_{1}(\gamma) n^{-1 / 2} e^{-\varkappa \gamma n} \exp \left\{-\frac{1}{2} \frac{\left(k-t \gamma^{-1} n\right)^{2}}{\sigma^{2}(\varkappa) \gamma^{-2} n}\right\}
$$

Суммируя (4.29) по области (I), приходим к требуемому заключению.

Выбирая $\delta=\delta_{n} \rightarrow 0$ так, что $\delta_{n} \ln n \rightarrow \infty$, мы обеспечим малость вклада членов (4.28). При условии $n^{\delta_{n}} / \ln n>A$ имеем (см. (4.27)):

$$
\exp \left\{-g\left(t, \frac{k}{n}\right)\right\} \leqslant e^{-\varkappa t n} \exp \left\{-\frac{1}{2}(b(\gamma, 1) A+o(1)) \ln n\right\},
$$

так что при $A>2 b^{-1}(\gamma, 1)=2 \sigma(\varkappa) \gamma^{-1}$ часть (III) пренебрежимо мала.

4.4. Доказательство теоремы 4.5. Сумму (1.3) разобьем на части по следующим областям:

$$
\text { (I) } k \geqslant n-n^{5 / 8}, \quad \text { (II) }(1-\varepsilon) n<k<n-n^{5 / 8}, \quad \text { (III) } k<(1-\varepsilon) n,
$$

где $\varepsilon \in\left(0,1-\beta \mu_{+}^{-1}\right)$. Для оценивания суммы по области (I) функцию $g(t, u)$ разложим по формуле Тейлора в точке $u=1$ :

$$
g(t, u)=\psi(t)+\ln \theta\left(\lambda_{t}\right) \cdot(1-u)+\frac{1}{2} b^{2}(t, 1)(1-u)^{2}+O(1)(1-u)^{3} .
$$


Преобразуем суммы средних членов в (4.30), выделив полный квадрат:

$$
\frac{1}{2} b^{2}(t, 1)\left(1-u+b^{-2}(t, 1) \ln \theta\left(\lambda_{t}\right)\right)^{2}-\frac{1}{2} b^{-2}(t, 1)\left(\ln \theta\left(\lambda_{t}\right)\right)^{2} .
$$

Подставим (4.31) в (4.30) и положим $u=k / n$. Полученное выражение для $g(t, k / n)$ подставим в формулу (3.6). Поскольку остаточный член в (4.30) при этом имеет порядок $n^{-9 / 8}$, то сумма по области (I) эквивалентна произведению $c_{1}(t) n^{-1 / 2} e^{-\psi(t) n}$ на выражение

$$
\exp \left\{\frac{\left(\ln \theta\left(\lambda_{t}\right)\right)^{2} n}{2 b^{2}(t, 1)}\right\} \sum_{0 \leqslant l \leqslant n-1} \exp \left\{-\frac{\left(l+b^{-2}(t, 1) \ln \theta\left(\lambda_{t}\right) n\right)^{2}}{2 b^{-2}(t, 1) n}\right\}
$$

что и дает правую часть в (4.10).

Для рассматриваемой области изменения $t$ функция $g(t, u)$ убывает по $u$, так что при $k$ из области (II) получаем

$$
\begin{gathered}
g\left(t, \frac{k}{n}\right) \geqslant g\left(t, 1-n^{-3 / 8}\right)=\psi(t)+\ln \theta\left(\lambda_{t}\right) \cdot n^{-3 / 8}+\frac{1}{2}\left(b^{2}(t, 1)+o(1)\right) n^{-6 / 8}, \\
\exp \left\{-g\left(t, \frac{k}{n}\right) n\right\} \leqslant e^{-\psi(t) n} \exp \left\{-\frac{1}{2}\left(b^{2}(t, 1)+o(1)\right) n^{1 / 4}\right\},
\end{gathered}
$$

т.е. сумма по области (II) пренебрежима мала. Для части (III) проходит оценка из доказательства теоремы 4.3. Теорема 4.5 доказана.

5. Асимптотика вероятностей больших уклонений максимума отрезка процесса ожидания и локальные предельные теоремы для момента его достижения. Следующие теоремы 5.1, 5.2 лежат в основе анализа асимптотики вероятностей больших уклонений максимума процесса ожидания

$$
\mathscr{M}_{n}^{W}:=\max \left(W_{j}: j \leqslant n\right) .
$$

Теорема 5.1. При условии (A1) равномерно по $t$ из любого отрезка $[\alpha, \beta] \subseteq\left(\mu_{0}^{+}, \mu_{+}\right)$

$$
\begin{aligned}
& \mathbf{P}\left(T(t n)=n, L_{1 n}>0\right) \sim \mathbf{P}\left(L_{1}^{(t)}>0\right) \mathbf{P}(T(t n)=n), \\
& \mathbf{P}\left(L_{1}^{(t)}>0\right)=\exp \left\{-\sum_{n=1}^{\infty} \frac{1}{n} \mathbf{P}\left(S_{n}^{(t)} \leqslant 0\right)\right\} .
\end{aligned}
$$

3 а м е ч а н и е. Соотношение (5.2) является аналогом следующего утверждения (см. [1, лемма 5]): $\mathbf{P}\left(S_{n}>t n, L_{1 n} \geqslant x\right) \sim \mathbf{P}\left(L_{1}^{(t)} \geqslant x\right) \mathbf{P}\left(S_{n}>\right.$ $t n)$. Соотношение $(5.2)$ верно и при замене знака $>$ в обоих местах на $\geqslant$.

Следствие 5.1. Равномерно по $t, k, n$ таким, ито $t n / k \in[\alpha, \beta] \subseteq$ $\left(\mu_{0}^{+}, \mu_{+}\right)$,

$$
\mathbf{P}\left(T(t n)=k, L_{1 k}>0\right) \sim \mathbf{P}\left(L_{1}^{(t n / k)}>0\right) \mathbf{P}(T(t n)=k)
$$


nрu $k, n \rightarrow \infty$.

Теорема 5.2. При $\mu_{0}<0$ равномерно по $t \in[\alpha, \beta] \subseteq\left(0, \mu_{+}\right)$

$$
\mathbf{P}\left(M_{n}>t n, L_{1, T(t n)}>0\right) \sim \mathbf{P}\left(L_{1}^{(\min (t, \gamma))}>0\right) \mathbf{P}\left(M_{n}>t n\right) .
$$

3 а м е ч а н и е. Соотношение (5.5) сохраняется, если при $t \in$ $\left(\gamma, \mu_{+}\right)$заменить там событие $\left\{L_{1, T(t n)}>0\right\}$ на $\left\{L_{1, n}>0\right\}$.

При $\mu_{0}<0$ введем процесс восстановления $\tau_{j}, j=1,2, \ldots$, образованный слабыми нижними лестничными моментами случайного блуждания $S_{n}$ (см. [3]) и положим $\tau_{0} \equiv 0$. Обозначим через $H(n):=\sum_{j=0}^{\infty} \mathbf{P}\left(\tau_{j} \leqslant n\right)$, $n=0,1,2, \ldots$, соответствующую функцию восстановления и определим $h_{n}:=H(n)-H(n-1) \equiv \mathbf{P}\left(\tau_{j}=n\right.$ при некотором $\left.j\right), h_{0}=1$.

Теорема 5.3. При $\mu_{0}<0$ равномерно по $t \in[\alpha, \beta] \subseteq\left(\gamma, \mu_{+}\right)$

$$
\begin{aligned}
\mathbf{P}\left(\mathscr{M}_{n}^{W}>t n\right) & \sim c_{2}(t) \mathbf{P}\left(L_{1}^{(t)}>0\right) \mathbf{P}\left(M_{n}>t n\right), \\
c_{2}(t):=\sum_{j=0}^{\infty} h_{j} \theta^{-j}\left(\lambda_{t}\right) & =1+\exp \left\{\sum_{n=1}^{\infty} \frac{\theta^{-n}\left(\lambda_{t}\right)}{n} \mathbf{P}\left(S_{n}<0\right)\right\} .
\end{aligned}
$$

Теорема 5.4. При $\mu_{0}<0$ равномерно по $t \in[\alpha, \beta] \subseteq(0, \gamma)$

$$
\begin{aligned}
\mathbf{P}\left(\mathscr{M}_{n}^{W}>t n\right) & \sim\left(\mathbf{E} \tau_{1}\right)^{-1} \mathbf{P}\left(L_{1}^{(\gamma)}>0\right)\left(1-t \gamma^{-1}\right) n \mathbf{P}\left(M_{n}>t n\right), \\
\mathbf{E} \tau_{1} & =\exp \left\{\sum_{n=1}^{\infty} \frac{1}{n} \mathbf{P}\left(S_{n}>0\right)\right\} .
\end{aligned}
$$

Теорема 5.5. При $\mu_{0}<0 u n \rightarrow \infty$

$$
\mathbf{P}\left(\mathscr{M}_{n}^{W}>\gamma n\right) \sim(2 \pi)^{-1 / 2}\left(\mathbf{E} \tau_{1}\right)^{-1} b^{-1}(\gamma, 1) \mathbf{P}\left(L_{1}^{(\gamma)}>0\right) \sqrt{n} \gamma^{-1} a(\gamma) e^{-\kappa \gamma n} .
$$

Отметим, что $\mathbf{P}\left(M_{n} \geqslant \gamma n\right) \sim(2 \gamma)^{-1} a(\gamma) e^{-\varkappa \gamma n}$ (см. теорему 4.4(ii)).

Пусть $Y$ - произвольный случайный вектор, $A-$ некоторое измеримое подмножество его значений, $P_{Y}(A)>0$. Обозначим через $(Y \mid A)$ случайный вектор с распределением $P_{(Y \mid A)}(B)=P_{Y}(B A) / P_{Y}(A)$; нам будет удобно считать, что с.в. $(Y \mid A)$ определена на основном вероятностном пространстве.

Определим случайные моменты: $\mathscr{T}(x)$ - первое пересечение процессом ожидания уровня $x>0, \mathscr{T}_{0}(x)-$ ближайший слева к $\mathscr{T}(x)$ нуль процесса ожидания, так что $0<W_{k}<x$ при $k \in\left(\mathscr{T}_{0}(x), \mathscr{T}\right)$, $W_{k}(x)<x$ при $0<k<\mathscr{T}_{0}(x), W_{\mathscr{T}_{0}(x)}=0, W_{\mathscr{T}(x)}>x$. Просматривая доказательства теорем 5.3-5.5, нетрудно прийти к следующим выводам.

Теорема 5.6. (i) $\Pi p u t \in\left(\gamma, \mu_{+}\right), m=0,1,2, \ldots$

$$
\mathbf{P}\left(\mathscr{T}_{0}(t n)=m \mid \mathscr{M}_{n}^{W}>t n\right) \longrightarrow \frac{h_{m} \theta^{-m}\left(\lambda_{t}\right)}{\sum_{j \geqslant 0} h_{j} \theta^{-j}\left(\lambda_{t}\right)}, \quad n \rightarrow \infty .
$$


(ii) При $t \in(0, \gamma)$ равномерно по $m \leqslant\left(1-t \gamma^{-1}\right) n-n^{1 / 2+\delta}, \delta>0$, $m, n \rightarrow \infty$,

$$
\mathbf{P}\left(\mathscr{T}_{0}(t n)=m \mid \mathscr{M}_{n}^{W}>t n\right) \sim\left(\left(1-t \gamma^{-1}\right) n\right)^{-1} .
$$

(iii) При $m, n \rightarrow \infty$ равномерно по $m=o\left(n^{2 / 3}\right)$

$$
\mathbf{P}\left(\mathscr{T}_{0}(\gamma n)=m \mid \mathscr{M}_{n}^{W}>\gamma n\right) \sim \frac{b(\gamma, 1)}{\sqrt{n}} \sqrt{2 \pi} \Phi\left(-\frac{b(\gamma, 1) m}{\sqrt{n}}\right) .
$$

(iv) $\Pi p u t \in\left(\gamma, \mu_{+}\right)$c.в. $\left(\mathscr{T}_{0}(t n) \mid \mathscr{M}_{n}^{W}>t n\right) u\left(n-\mathscr{T}(t n) \mid \mathscr{M}_{n}^{W}>t n\right)$ асимптотически независимь при $n \rightarrow \infty u$

$\mathbf{P}\left(n-\mathscr{T}(t n)=k \mid \mathscr{M}_{n}^{W}>t n\right) \longrightarrow \theta^{-k}\left(\lambda_{t}\right)\left(1-\theta^{-1}\left(\lambda_{t}\right)\right), \quad k=0,1,2, \ldots$

(v) $\Pi p u t \in(0, \gamma)$ c.в.

$$
\left(\frac{\mathscr{T}_{0}(t n)}{\left(1-t \gamma^{-1}\right) n} \mid \mathscr{M}_{n}^{W}>t n\right) \quad u \quad\left(\frac{\mathscr{T}(t n)-\left(\mathscr{T}_{0}(t n)+t \gamma^{-1} n\right)}{b^{-1}\left(t, t \gamma^{-1}\right) \sqrt{n}} \mid \mathscr{M}_{n}^{W}>t n\right)
$$

асимптотически независимь при $n \rightarrow \infty$, предельное распределение первой - равномерное на $[0,1]$, а второй - стандартное нормальное.

(vi) При $m, l, n \rightarrow \infty$ равномерно по $m, l=o\left(n^{2 / 3}\right)$

$$
\begin{aligned}
\mathbf{P}\left(\mathscr{T}_{0}(\gamma n)=m, n-\right. & \left.\mathscr{T}(\gamma n)=l \mid \mathscr{M}_{n}^{W}>\gamma n\right) \\
& \sim \sqrt{2 \pi} \frac{b^{2}(\gamma, 1)}{n} \varphi\left(\frac{m+l}{b^{-1}(\gamma, 1) \sqrt{n}}\right), \\
\mathbf{P}\left(n-\mathscr{T}(\gamma n)=l \mid \mathscr{T}_{0}(\gamma n)=m, \mathscr{M}_{n}^{W}>\gamma n\right) & \sim \frac{b(\gamma, 1) \varphi\left((m+l) b(\gamma, 1) n^{-1 / 2}\right)}{\sqrt{n} \Phi\left(-b(\gamma, 1) m n^{-1 / 2}\right)} .
\end{aligned}
$$

5.1. Доказательство теоремы 5.1. Следующий аналог теоремы 3 работы [7] включает в себя утверждение (5.2).

Предложение 5.1. Пусть $B$ - измеримое множество в пространстве $\mathbf{R} \times \mathbf{Z}_{+}^{\infty}=\left\{\left(x, i_{1}, i_{2}, \ldots\right): x-\right.$ действительное, $i_{k}-$ натуральнье $\}, B_{k}-$ сечение множества $B$ координатой $i_{k}$. Предположим, что

$$
B_{k} \subseteq \overline{[(t-\delta) k,(t+\delta) k]} \quad \text { при } k \geqslant N \text { и некотором } \delta>0,
$$

вьполнено условие (A1) $u t \in\left(\mu_{0}^{+}, \mu_{+}\right)$. Тогда

$$
\mathbf{P}\left(T(t n)=n, S_{j} \notin B_{j}, j \leqslant n\right) \sim \mathbf{P}\left(S_{j}^{(t)} \notin B_{j}, j \geqslant 1\right) \mathbf{P}(T(t n)=n) .
$$

Соотношение (5.12) выполняется равномерно по $t \in[\alpha, \beta] \subseteq\left(\mu_{0}^{+}, \mu_{+}\right)$в предположении, что (5.11) выполняется для $\delta<\min \left(\alpha-\mu_{0}^{+}, \mu_{+}-\beta\right) u$ для $N$, не зависящего от $t$. 
3 а м е ч а н и е. В [7] вместо события $\{T(t n)=n\}=\left\{S_{n}>t n\right.$, $\left.M_{n-1} \leqslant t n\right\}$ в (5.12) взято (фактически) событие $S_{n} \in[t n, t n+\Delta]$, наше рассуждение сохраняет силу и для условия $S_{n} \in[t n, t n+\Delta], M_{n-1} \leqslant t n$.

Док аз ательс тво пр д ло жения 5.1. Шаг 1. Проверим, что при любом $k$

$$
\left(X_{1}, X_{2}, \ldots, X_{k} \mid T(t n)=n\right) \stackrel{d}{\longrightarrow}\left(X_{1}^{(t)}, X_{2}^{(t)}, \ldots, X_{k}^{(t)}\right) .
$$

Вероятность $\mathbf{P}\left(X_{i} \in\left[a_{i}, b_{i}\right], i \leqslant k, T(t n)=n\right)$ запишем в виде

$$
\begin{aligned}
& \int_{\left\{x_{i} \in\left[a_{i}, b_{i}\right], i \leqslant k, m_{n-1} \leqslant t n, s_{n}>t n\right\}} \ldots \prod_{i=1}^{n} d F\left(x_{i}\right) \\
= & \int_{\left\{x_{i} \in\left[a_{i}, b_{i}\right], i \leqslant k\right\}} \ldots \int_{i} \mathbf{P}\left(s_{k}+\widetilde{M}_{n-k-1} \leqslant t n, s_{k}+\widetilde{S}_{n-k}>t n\right) \prod_{i=1}^{k} d F\left(x_{i}\right),
\end{aligned}
$$

где $\widetilde{S}_{n-k}:=S_{n}-S_{k}, \widetilde{M}_{n-k-1}:=\max \left(S_{j}-S_{k}: k<j<n\right)$ и предполагается, что $\max \left(b_{1}+\cdots+b_{j}: j \leqslant k\right) \leqslant t n$. Вероятность под знаком интеграла в (5.14) эквивалентна (равномерно по $x_{i}$ и $t$ ) выражению (см. (3.3)):

$$
c_{1}(t) n^{-1 / 2} \exp \left\{-\psi\left(\frac{t n-s_{k}}{n-k}\right)(n-k)\right\} .
$$

Применяя формулу Тейлора, имеем:

$$
\psi\left(t+\frac{t n-s_{k}}{n-k}\right)(n-k)=\psi(t) n+k \ln \theta\left(\lambda_{t}\right)-\lambda_{t} s_{k}+O\left(\frac{1}{n}\right) .
$$

Подставив (5.16) в (5.15), а результат - в (5.14), получим (5.13).

Шаг 2. Вероятность слева в (5.12) при любом $k<n$ не превосходит

$$
\mathbf{P}\left(T(t n)=n, S_{j} \notin B_{j}, j \leqslant k\right) .
$$

Ввиду (5.13) вероятность (5.17) эквивалентна при $n \rightarrow \infty$ выражению $\mathbf{P}\left(S_{j}^{(t)} \notin B_{j}, j \leqslant k\right) \mathbf{P}(T(t n)=n)$, откуда получаем:

$$
\varlimsup_{n \rightarrow \infty} \mathbf{P}\left(S_{j} \notin B_{j}, j \leqslant n \mid T(t n)=n\right) \geqslant \mathbf{P}\left(S_{j}^{(t)} \notin B_{j}, j \leqslant k\right) .
$$

Шаг 3. Оценим сверху вероятности

$$
\mathbf{P}\left(T(t n)=n, S_{k}>(t+\delta) k\right) \leqslant \mathbf{P}\left(S_{n}>t n, S_{k}>(t+\delta) k\right)
$$

при $k \leqslant n$. Применяя к вероятности справа в (5.19) преобразования начала п. 3.1, получим для нее выражение (ср. с (3.11)):

$$
e^{-\psi(t) n} \int_{\left\{s_{k}>(t+\delta) k, s_{n}>t n\right\}} \ldots \int^{-\lambda_{t}\left(s_{n}-t n\right)} \prod_{i=1}^{n} d F_{\lambda_{t}}\left(x_{i}\right) .
$$


Интеграл в (5.20) запишем в виде

$$
\begin{aligned}
& \mathbf{E}\left(e^{-\lambda_{t}\left(S_{n}^{(t)}-t n\right)} ; S_{k}^{(t)}>(t+\delta) k, S_{n}^{(t)}>t n\right) \\
& \quad=\int_{\delta k}^{\infty} \mathbf{E}\left(e^{-\lambda_{t}\left(S_{n-k}^{(t)}-t(n-k)+x\right)} ; S_{n-k}^{(t)}-t(n-k)+x>0\right) \mathbf{P}\left(S_{k}^{(t)}-t k \in d x\right) .
\end{aligned}
$$

При $k>A \ln n$ оценим (5.21) сверху вероятностью (см. (1.10))

$$
\mathbf{P}\left(S_{k}^{(t)}>(t+\delta) k\right) \leqslant \exp \left\{-\psi^{(t)}(t+\delta) k\right\} \leqslant n^{-A \psi^{(t)}(t+\delta)} .
$$

Выбирая $A$ из условия $A \psi^{(t)}(t+\delta) \geqslant 2$, получим для вероятностей (5.19) при $k>A \ln n$ оценку сверху

$$
n^{-2} e^{-\psi(t) n}
$$

При $k \leqslant A \ln n$ разобьем область интегрирования в (5.21) на части $(\delta k, \sqrt[3]{n})$ и $[\sqrt[3]{n}, \infty)$. Интеграл $(5.21)$ по области $[\sqrt[3]{n}, \infty)$ оценим с помощью первого неравенства в (5.22) и получим для него границу сверху

$$
e^{-\psi^{(t)}(t+\delta) \sqrt[3]{n}} e^{-\psi(t) n}
$$

Интеграл (5.21) по области $(\delta k, \sqrt[3]{n}), k \leqslant A \ln n$, не превосходит

$\mathbf{P}\left(S_{k}^{(t)}>(t+\delta) k\right) \max \left\{\mathbf{E}\left(e^{-\lambda_{t}\left(S_{m}^{(t)}-t m+x\right)} ; S_{m}^{(t)}-t m+x>0\right): \delta k \leqslant x \leqslant \sqrt[3]{n}\right)$,

где для краткости положено $m:=n-k$. К вероятности в (5.25) применим первое неравенство в (5.22). Математическое ожидание в (5.25) запишем в интегральной форме и получим:

$$
\int_{-x}^{\infty} e^{-\lambda_{t}(y+x)} \mathbf{P}\left(S_{m}^{(t)}-t m \in d y\right) \sim \frac{1}{\lambda_{t} \sigma\left(\lambda_{t}\right) \sqrt{2 \pi n}}
$$

равномерно по $|x| \leqslant \sqrt[3]{n}$ (ср. с оценкой интеграла (3.13)). Отсюда для интеграла $(5.21)$ по области $(\delta k, \sqrt[3]{n})$ получаем при всех достаточно больших $n$ оценку сверху

$$
(1+\varepsilon) e^{-\psi^{(t)}(t+\delta) k}\left(\lambda_{t} \sigma\left(\lambda_{t}\right) \sqrt{2 \pi n}\right)^{-1} .
$$

Таким образом, при $k \leqslant A \ln n$ верхняя граница для (5.19) есть сумма выражения (5.24) и выражения (5.26), домноженного на $e^{-\psi(t) n}$.

Шаг 4. К вероятностям

$$
\mathbf{P}\left(T(t n)=n, S_{k}<(t-\delta) k\right) \leqslant \mathbf{P}\left(S_{n}>t n, S_{k}<(t-\delta) k\right)
$$

применим построения шага 3 , в результате чего для них сохранятся оценки $(5.23),(5.24)$ с заменой $\psi^{(t)}(t+\delta)$ на $\psi^{(t)}(t-\delta)$ и с такой же заменой - оценка (5.26) с множителем $e^{-\psi(t) n}$. 
ШІаг 5. Оценим сверху вероятность

$$
\begin{gathered}
\mathbf{P}\left(T(t n)=n, S_{j} \notin B_{j}, j \leqslant N, \bigcup_{k=N+1}^{n}\left\{S_{k} \in B_{k}\right\}\right) \\
\leqslant \sum_{k=N+1}^{n} \mathbf{P}\left(T(t n)=n,\left|S_{k}-t k\right|>\delta k\right),
\end{gathered}
$$

применяя к слагаемым суммы (5.28) оценки шагов 3,4 . Положим для краткости $a_{\delta}^{(t)}:=\min \left(\psi^{(t)}(t+\delta), \psi^{(t)}(t-\delta)\right)$. Сумма $(5.28)$ при $k \leqslant A \ln n$ оценивается сверху для достаточно больших $n$ выражением

$2\left(A \ln n \cdot e^{-a_{\delta}^{(t)} \sqrt[3]{n}}+\frac{(1+\varepsilon) c_{\delta}^{(t)}}{\lambda_{t} \sigma\left(\lambda_{t}\right) \sqrt{2 \pi n}} e^{-a_{\delta}^{(t)} N}\right) e^{-\psi(t) n}, \quad c_{\delta}^{(t)}:=\left(1-e^{-a_{\delta}^{(t)}}\right)^{-1}$,

а при $k>A \ln n$, благодаря (5.23), - величиной $n^{-1} e^{-\psi(t) n}$.

Шаг 6. Выпишем для вероятности в левой части (5.12) оценку снизу:

$$
\mathbf{P}\left(T(t n)=n, S_{j} \notin B_{j}, j \leqslant N\right)-\mathbf{P}\left(T(t n)=n, \bigcup_{j=N+1}^{n}\left\{S_{j} \in B_{j}\right\}\right) .
$$

Подставив оценки шага 5 в $(5.29)$ и поделив на $\mathbf{P}(T(t n)=n)$, получим:

$$
\begin{aligned}
& \varliminf_{n \rightarrow \infty} \mathbf{P}\left(S_{j} \notin B_{j}, j \leqslant n \mid T(t n)=n\right) \\
& \quad \geqslant \mathbf{P}\left(S_{j}^{(t)} \in B_{j}, j \leqslant N\right)-\frac{2(1+\varepsilon) c_{\delta}^{(t)}}{\lambda_{t} a(t)} e^{-a_{\delta}^{(t)} N} .
\end{aligned}
$$

Переход к пределу в (5.18), (5.30) при $k, N \rightarrow \infty$ приводит к соотношению (5.12). Предложение 5.1 доказано.

Соотношение (5.2) теоремы 5.1 получается подстановкой в (5.12) $B_{j}=(-\infty, 0]$. Равенство (5.3) вытекает из тождества Спарре-Андерсена (см. [3]).

5.2. Доказательство теоремы 5.2. Ход рассуждений лишь в деталях отличается от доказательств соответствующих утверждений пп. 4.3, 4.4. Вывод соотношения (5.5) опирается на разложение

$$
\mathbf{P}\left(M_{n}>t n, L_{1, T(t n)}>0\right)=\sum_{k=1}^{n} \mathbf{P}\left(T(t n)=k, L_{1 k}>0\right),
$$

заменяющее (1.3). $\mathrm{K}$ членам суммы (5.31) применяется асимптотика (5.4), лемма 2.1(ii) и оценки сверху теорем 4.4(ii), 4.5. Отдельно рассматриваются случаи $t \in[\alpha, \gamma]$ и $t \in[\gamma, \beta]$. В первом случае область суммирования в (5.31) разбивается на части (I)-(IV) из п. 4.3. Слагаемые суммы по области (I) оцениваются с помощью соотношения (5.4), а оценка для сумм по областям (II)-(IV) следует из оценок теоремы 4.4 
и неравенства $\mathbf{P}\left(T(t n)=k, L_{1 k}>0\right) \leqslant \mathbf{P}(T(t n)=k)$. По той же схеме проводится доказательство теоремы 5.2 для $t \in[\gamma, \beta)$, но со ссылкой на п. 4.4 .

5.3. Доказательство теорем 5.3-5.5. Введем обозначения

$$
\begin{aligned}
T_{j}(x) & :=\inf \left(k>j: S_{k}-S_{j}>x\right), \quad j=0,1,2, \ldots, \quad T_{0}(x) \equiv T(x), \\
M_{j n} & :=\max \left(S_{k}-S_{j}: j \leqslant k \leqslant n\right), \quad M_{0 n} \equiv M_{n}, \\
L_{j+1, n} & :=\min \left(S_{k}-S_{j}: j+1 \leqslant k \leqslant n\right), \quad j \geqslant 0, \\
B_{m n}^{(t n)} & :=\left\{\tau_{j}=m \text { при некотором } j, \quad M_{m n}>t n, L_{m+1, T_{m}(t n)}>0\right\}
\end{aligned}
$$

и заметим, что

$$
\begin{gathered}
\left\{\mathscr{M}_{n}^{W}>t n\right\}=\bigcup_{m<n} B_{m n}^{(t n)}, \\
\mathbf{P}\left(B_{m n}^{(t n)}\right)=h_{m} \mathbf{P}\left(M_{m n}>t n, L_{m+1, T_{m}(t n)}>0\right), \\
\mathbf{P}\left(B_{m n}^{(t n)}\right) \leqslant P\left(M_{n-m}>t n\right)=\sum_{k=1}^{n-m} \mathbf{P}(T(t n)=k) .
\end{gathered}
$$

(i) Сумма в (5.34) есть часть (при $m>0$ ) суммы в (1.3), слагаемые последней оценивались в пп. 4.1-4.3. При $t n / k \in[\alpha, \beta] \subseteq\left(0, \mu_{+}\right)$асимптотика слагаемых дается соотношением (3.6), при этом экспоненциальный сомножитель в асимптотике есть $\exp \{-g(t, k / n) n\}$ (см. (3.19)). Для $t>\gamma$ асимптотику суммы (1.3) определяют ее члены с $k$ вблизи $n$, а экспоненциальная составляющая их асимптотики равна $\exp \{-g(t, 1) n\} \equiv$ $\exp \{-\psi(t) n\}$. Для $t \leqslant \gamma$ определяющими являются значения $k / n \approx t \gamma^{-1}$, соответствующая экспонента есть $\exp \{-\varkappa t n\}$. Оценки периферийных членов суммы (1.3), полученные в пп. 4.1-4.3, добавляют к экспоненциальному члену асимптотики множитель, убывающий как экспонента от $n^{a}, a>0$. Указанные соображения приводят к заключению, что в условиях теоремы 5.3 члены объединения (5.32) с $m>A \ln n$ могут быть при подходящем выборе постоянной $A>0$ отброшены без нарушения объявленной асимптотики вероятностей больших уклонений максимума (5.6). Аналогичные соображения будут использованы в доказательстве теоремы 5.4 .

В объединении (5.32) оставим члены с $m \leqslant A \ln n$ и воспользуемся неравенствами

$$
\sum_{m} \mathbf{P}\left(B_{m n}^{(t n)}\right)-\sum_{l<m} \mathbf{P}\left(B_{l n}^{(t n)} B_{m n}^{(t n)}\right) \leqslant \mathbf{P}\left(\bigcup_{m} B_{m n}^{(t n)}\right) \leqslant \sum_{m} P\left(B_{m n}^{(t n)}\right),
$$

где суммирование ведется по $m, l \leqslant A \ln n$. Из (5.33), (5.5) получаем:

$$
\mathbf{P}\left(B_{m n}^{(t n)}\right) \sim h_{m} \mathbf{P}\left(L_{1}^{(t)}>0\right) \mathbf{P}\left(M_{n-m}>t n\right)
$$


равномерно по указанным $m$. Из (4.1) следует, что

$$
\mathbf{P}\left(M_{n-m}>t n\right) \sim\left(1-\theta^{-1}\left(\lambda_{t}\right)\right)^{-1} c_{1}(t) n^{-1 / 2} \exp \left\{-\psi\left(\frac{t n}{n-m}\right)(n-m)\right\},
$$

также равномерно по $m$. Подставляя $(5.37)$ в (5.36), суммируя по $m$ и применяя разложение функции $\psi(t n /(n-m))$ по формуле Тейлора (см. (3.23), (3.24)), получаем для одинарной суммы в (5.35) асимптотически эквивалентное выражение, совпадающее с правой частью соотношения (5.6).

События $B_{m n}^{(t n)}$ и $B_{l n}^{(t n)}$ при $m-l \leqslant T_{l}(t n)$ несовместны. При $T_{l}(t n)<$ $m-l$ события $\left\{M_{l n}>t n, L_{l+1, T_{l}(t n)}>0\right\}$ и $\left\{M_{m n}>t n, L_{m+1, T_{m}(t n)}>0\right\}$ независимы. Из всего этого следует, что двойная сумма в (5.35) пренебрежимо мала, так что соотношение (5.6) установлено. Второе равенство в (5.7) есть следствие тождества Спарре-Андерсена [3].

(ii) Ход рассуждений в доказательстве теоремы 5.4 тот же, что и в теореме 5.3. В объединении (5.32) отбрасываем члены с $m>$ $\left(1-t \gamma^{-1}\right) n+n^{5 / 8}$. Оценка (4.27) показывает, что вероятности этих членов асимптотически не больше, чем $e^{-a \sqrt[4]{n}} e^{-\varkappa t n}$ при некотором $a>0$ и всех $t \in[\alpha, \beta]$, так что они не влияют на асимптотику вероятностей больших уклонений $\mathbf{P}\left(\mathscr{M}_{n}^{W}>t n\right)$. При $\left|m-\left(1-t \gamma^{-1}\right) n\right| \leqslant n^{5 / 8}$ из (5.34), (4.24), (4.25) следует, что $\mathbf{P}\left(B_{m n}^{(t n)}\right)=O(1) e^{-\varkappa t n}, n \rightarrow \infty$. Число таких членов не превосходит $2 n^{5 / 8}$, и ими также пренебрегаем. Применяя (5.36) и теорему 4.4(i), получаем:

$$
\mathbf{P}\left(B_{m n}^{(t n)}\right) \sim h_{m} \mathbf{P}\left(L_{1}^{(t)}>0\right) \gamma^{-1} a(\gamma) e^{-\varkappa t n}
$$

равномерно по $m \leqslant\left(1-t \gamma^{-1}\right) n-n^{5 / 8}$ и по указанным $t$. Суммируя соотношения (5.38), приходим к правой части (5.8). Двойной суммой в (5.35) можно пренебречь по тем же соображениям, что и в теореме 5.3. Соотношение (5.9) есть следствие тождества Спарре-Андерсена [3]. Теорема 5.4 доказана.

(iii) Доказательство теоремы 5.5 проводится по уже использованной выше схеме. В объединении (5.32) мы оставляем члены с $m \leqslant n^{5 / 8}$. Соображения, использованные в теореме 5.4 , показывают, что членами с $m>n^{5 / 8}$ можно пренебречь. При $m \leqslant n^{5 / 8}$, применяя теорему 4.4(ii), (iii), получаем, что при $n \rightarrow \infty$ равномерно по $m$

$$
\mathbf{P}\left(B_{m n}^{(\gamma n)}\right) \sim h_{m} \mathbf{P}\left(L_{1}^{(\gamma)}>0\right) \Phi\left(\sqrt{n-m} b(\gamma, 1)\left(1-\frac{n}{n-m}\right)\right) \gamma^{-1} a(\gamma) e^{-\varkappa \gamma n} .
$$

Суммируя эти соотношения по $m \leqslant n^{5 / 8}$, получаем:

$$
\mathbf{P}\left(\mathscr{M}_{n}^{W}>\gamma n\right) \sim \mathbf{P}\left(L_{1}^{(\gamma)}>0\right) \gamma^{-1} a(\gamma) e^{-\varkappa \gamma n} \sum_{m \leqslant n^{5 / 8}} \Phi\left(-\frac{b(\gamma, 1) m}{\sqrt{n-m}}\right) h_{m} .
$$


Заменяя последнюю сумму на эквивалентное при $n \rightarrow \infty$ выражение

$$
\left(\mathbf{E} \tau_{1}\right)^{-1} b^{-1}(\gamma, 1) \sqrt{n} \int_{-\infty}^{0} \Phi(x) d x=\left(\mathbf{E} \tau_{1}\right)^{-1} b^{-1}(\gamma, 1) \sqrt{n}(2 \pi)^{-1 / 2},
$$

приходим к (5.10). Теорема 5.5 доказана.

6. Условные предельные теоремы для отрезка случайного блуждания и процесса ожидания до первого момента пересечения высокого уровня. В пространстве $C[0,1]$ непрерывных функций на отрезке $[0,1]$ с равномерной метрикой введем случайный элемент $Y_{m}^{(x)}(\cdot), x>0, m-$ натуральное, полагая $Y_{m}^{(x)}(s):=\left(S_{s m}-\right.$ $s x) /(\sigma(\lambda(x / m)) \sqrt{m})$ при $0 \leqslant s \leqslant 1$ таких, что число $s m-$ целое, и доопределяя величины $Y_{m}^{(x)}(s)$ при остальных $s$ линейной интерполяцией. Для любого случайного элемента $Y(\cdot) \in C[0,1]$ и любого измеримого подмножества $A \subseteq C[0,1]$ введем обозначение $(Y(\cdot) \mid A)$ для случайного элемента из $C[0,1]$ с распределением вероятностей $P_{(Y(\cdot) \mid A)}(B):=$ $P_{Y(\cdot)}(B A) / P_{Y(\cdot)}(A), P_{Y(\cdot)}(A)>0$, являющимся условным распределением $Y(\cdot)$ относительно $A$.

Ниже мы опираемся на следующий известный результат.

Теорема 6.1 (см. [7]-[9]). Пусть выполнено условие (A1), тогда npu $t \in\left(\mu_{0}, \mu_{+}\right)$

$$
\left(Y_{n}^{(t n)}(\cdot) \mid S_{n}>t n\right) \stackrel{d}{\longrightarrow} w_{0}(\cdot) .
$$

Дополнение к теореме 6.1. Равномерно по $t=t_{m n}$, изменяюиимся так, ито $t n / m \in[\alpha, \beta] \subseteq\left(\mu_{0}^{+}, \mu_{+}\right)$,

$$
\left(Y_{m}^{(t n)}(\cdot) \mid S_{m}>t n\right) \stackrel{d}{\longrightarrow} w_{0}(\cdot), \quad m, n \rightarrow \infty .
$$

З а м е ч а н и е. Вывод (6.1) в [7]-[9] проводится в два этапа: доказательство сходимости конечномерных распределений и доказательство плотности. В [7], [8] постановка задачи более общая: многомерный случай и условие $S_{n} \in[x, x+\Delta]$ при любых $x$ и $\Delta$, кроме того там принято дополнительно условие строгой нерешетчатости распределения с.в. $X$, в нашем случае излишнее. Ради целостности изложения, а также ввиду необходимой нам равномерности по $t$, указанной выше, мы приводим краткий вывод (6.1), основанный на теореме 24.2 книги [10] о сходимости к броуновскому мосту в случае симметрично зависимых величин.

Теорема 6.2. Для любого фиксированного $t \in\left(\mu_{0}^{+}, \mu_{+}\right)$имеет место следующая сходимость по распределению случайных элементов пространства $C[0,1] \kappa$ броуновскому мосту:

$$
\left(Y_{n}^{(t n)}(\cdot) \mid T(t n)=n\right) \stackrel{d}{\longrightarrow} w_{0}(\cdot) .
$$

Теорема 6.3. Утверждение (6.2) остается в силе, если $t$ изменяется с ростом $n$ в пределах любого отрезка $[\alpha, \beta] \subseteq\left(\mu_{0}^{+}, \mu_{+}\right)$. 
Следствие 6.1. При $t, m, n$ maкux, чmo $t n / m \in[\alpha, \beta] \subseteq\left(\mu_{0}^{+}, \mu_{+}\right)$,

$$
\left(Y_{m}^{(t n)}(\cdot) \mid T(t n)=m\right) \stackrel{d}{\longrightarrow} w_{0}(\cdot), \quad m, n \rightarrow \infty .
$$

Следствие 6.2. При $t, m, n$ maкux, чmo $t n / m \in[\alpha, \beta] \subseteq\left(\mu_{0}^{+}, \mu_{+}\right)$,

$$
\left(Y_{m}^{(t n)}(\cdot) \mid T(t n)=m, L_{1 m}>0\right) \stackrel{d}{\longrightarrow} w_{0}(\cdot), \quad m, n \rightarrow \infty .
$$

При заданном $n$ от блуждания $S_{j}=\sum_{i=1}^{j} X_{i}, 0 \leqslant j \leqslant n, S_{0}=0$, перейдем к обращенному блужданию $\widehat{S}_{k}:=S_{n}-S_{n-k}, 0 \leqslant k \leqslant n$, и пусть $\widehat{T}(t n), t>0$, обозначает момент его первого выхода за уровень $t n$. В системе координат, связанной с обращенным блужданием (в частности, обращенным временем), отрезок блуждания после момента $\widehat{T}(t n)$ представляет собой независимую копию исходного блуждания $S_{j}$ (с началом в точке $\widehat{S}_{\widehat{T}(t n)}$ и случайной длительности $\left.n-\widehat{T}(t n)\right)$. Таким образом, получаем следующую теорему.

Теорема 6.4. При условии $\widehat{T}(t n)=k, 0<k<n$, траектория прочесса ожидания $W_{j}, j \leqslant n$, распадается на две условно независимье части: $W_{j}, j \leqslant n-k, u W_{n-k+i}-W_{n-k}, i \leqslant k$. При этом

$$
\begin{aligned}
\left(W_{j}, j \leqslant n-k \mid \widehat{T}(t n)\right. & =k) \stackrel{d}{=}\left(W_{j}, j \leqslant n-k \mid W_{n-k}=0\right), \\
\left(W_{n}-W_{i}, i \leqslant k \mid \widehat{T}(t n)\right. & =k) \stackrel{d}{=}\left(S_{i}, i \leqslant k \mid T(t n)=k\right), \\
\left(W_{n-k+i}-W_{n-k}, i \leqslant k \mid \widehat{T}(t n)\right. & =k) \stackrel{d}{=}\left(S_{i}, i \leqslant k \mid S_{k}>t n, L_{1 k}>0\right) .
\end{aligned}
$$

Соотношения (6.4) сводят описание условного асимптотического поведения процесса ожидания при условии больших уклонений $W_{n}>t n \mathrm{~K}$ уже изученным вопросам.

Изучение отдельных участков блуждания $S_{k}, k \leqslant n$, при условии события $M_{n}>t n$ содержится в диссертации А.В.Шкляева [11]. Мы дополним его исследование следующим утверждением.

Теорема 6.5. Пусть $t$ и $k$ изменяются при $n \rightarrow \infty$ так, что $t n / k \in[\alpha, \beta] \subseteq\left(\mu_{0}^{+}, \mu_{+}\right)$. Тогда при любом фиксированном $m$ имеет место сходимость по распределению случайных векторов:

$$
\begin{gathered}
\left(S_{T(t n)}-t n, S_{T(t n)}-S_{T(t n)-1}, \ldots, S_{T(t n)}-S_{T(t n)-m} \mid T(t n)=k\right) \\
\stackrel{d}{\longrightarrow}\left(Y_{0}^{(t)}, Y_{1}^{(t)}, \ldots, Y_{m}^{(t)}\right) .
\end{gathered}
$$

Вектор $\left(Y_{1}^{(t)}, Y_{2}^{(t)}, \ldots, Y_{n}^{(t)} \mid Y_{0}^{(t)}=y_{0}\right)$ представляет собой отрезок однородной марковской цепи с переходной вероятностью (см. (6.18)), зависящей от $y_{0}$ как от параметра, совместное распределение вероятностей с.в. $Y_{0}^{(t)}, Y_{1}^{(t)}, \ldots, Y_{n}^{(t)}$ является смесью условньх распределений с.в. $Y_{0}^{(t)}, Y_{1}^{(t)}, \ldots, Y_{n}^{(t)}$ при условии $Y_{0}^{(t)}=y_{0}$ (см. (6.19)). 
6.1. Доказательство теоремы 6.1. Пусть при каждом $n$ с.в. $\xi_{n 1}, \xi_{n 2}, \ldots, \xi_{n n}$ симметрично зависимы в том смысле, что их совместное распределение инвариантно относительно их перестановок. Введем случайный элемент $Y_{n}(\cdot)$ пространства $C[0,1]$, полагая $Y_{n}(s):=\sum_{i=1}^{s n} \xi_{n i}$ при $0 \leqslant s \leqslant 1$ таких, что число $s n$ целое, и доопределяя величины $Y_{n}(s)$ при остальных $s$ линейной интерполяцией. Воспользуемся следующим утверждением, вытекающим из теоремы 24.2 книги [10]: если

$$
\sum_{i=1}^{n} \xi_{n i} \stackrel{p}{\longrightarrow} 0, \quad \sum_{i=1}^{n} \xi_{n i}^{2} \stackrel{p}{\longrightarrow} 1, \quad \max _{1 \leqslant i \leqslant n}\left|\xi_{n i}\right| \stackrel{p}{\longrightarrow} 0 \quad \text { npu } n \rightarrow \infty,
$$

то имеет место сходимость $Y_{n}(\cdot) \stackrel{d}{\longrightarrow} w_{0}(\cdot)$ в $C[0,1]$. Положим

$$
\left(\xi_{n 1}, \ldots, \xi_{n n}\right):=\left(\frac{X_{1}-t}{\sigma\left(\lambda_{t}\right) \sqrt{n}}, \ldots, \frac{X_{n}-t}{\sigma\left(\lambda_{t}\right) \sqrt{n}} \mid S_{n}>t n\right) .
$$

Величины (6.7), очевидно, симметрично зависимы.

(i) Из теоремы В. В. Петрова (1.4) легко следует, что условное предельное распределение с.в. $S_{n}-t n$ при условии $S_{n}>t n$ является экспоненциальным с параметром $\lambda_{t}$. Отсюда и из соотношения $\left(S_{n}-t n \mid S_{n}>\right.$ $t n)=\sigma\left(\lambda_{t}\right) \sqrt{n} \sum_{i=1}^{n} \xi_{n i}$ следует первое соотношение в (6.6).

(ii) Вероятность $\mathbf{P}\left(\left|\sum_{i=1}^{n}\left(X_{i}-t\right)^{2} /\left(\sigma^{2}\left(\lambda_{t}\right) n\right)-1\right|>\varepsilon, S_{n}>t n\right)$ преобразуем по схеме п. 3.1 :

$$
\begin{aligned}
\int \cdots & \left.\int\left|\sum_{i=1}^{n} \frac{\left(x_{i}-t\right)^{2}}{\sigma^{2}\left(\lambda_{t}\right) n}-1\right|>\varepsilon, s_{n}>t n\right\} \prod_{i=1}^{n} d F\left(x_{i}\right) \\
= & e^{-\psi(t) n} \mathbf{E}\left\{e^{-\lambda_{t}\left(S_{n}^{(t)}-t n\right)} ;\left|\sum_{i=1}^{n} \frac{\left(X_{i}^{(t)}-t\right)^{2}}{\sigma^{2}\left(\lambda_{t}\right) n}-1\right|>\varepsilon, S_{n}^{(t)}>t n\right\} .
\end{aligned}
$$

Математическое ожидание в последнем выражении оценим сверху вероятностью $\mathbf{P}\left(\left|\sum_{i=1}^{n}\left(X_{i}^{(t)}-t\right)^{2} /\left(\sigma^{2}\left(\lambda_{t}\right) n\right)-1\right|>\varepsilon\right)$, не превосходящей по неравенству Чебышева $\varepsilon^{-2} n^{-1} \sigma^{-4}\left(\lambda_{t}\right) \mathbf{D}\left(X^{(t)}-t\right)^{2}$. Таким образом, при любом $\varepsilon>0$ имеем: $\mathbf{P}\left(\left|\sum_{i=1}^{n} \xi_{n i}^{2}-1\right|>\varepsilon\right)=O\left(n^{-1 / 2}\right), n \rightarrow \infty$.

(iii) Снова используя схему рассуждений п. 3.1 , получаем:

$$
\begin{aligned}
& \mathbf{P}\left(\max _{1 \leqslant i \leqslant n}\left|\xi_{n i}\right|>\varepsilon\right) \leqslant n \mathbf{P}\left(\left|\xi_{n 1}\right|>\varepsilon\right) \\
& \mathbf{P}\left(\frac{\left|X_{1}-t\right|}{\sigma\left(\lambda_{t}\right) \sqrt{n}}>\varepsilon, S_{n}>t n\right) \\
& \quad=e^{-\psi(t) n} \mathbf{E}\left\{e^{-\lambda_{t}\left(S_{n}^{(t)}-t n\right)} ; \frac{\left|X_{1}^{(t)}-t\right|}{\sigma\left(\lambda_{t}\right) \sqrt{n}}>\varepsilon, S_{n}^{(t)}>t n\right\} \\
& \quad \leqslant e^{-\psi(t) n} \mathbf{P}\left(\frac{\left|X_{1}^{(t)}-t\right|}{\sigma\left(\lambda_{t}\right) \sqrt{n}}>\varepsilon\right) .
\end{aligned}
$$


Случайная величина $X_{1}^{(t)}$ удовлетворяет двустороннему условию Крамера, поэтому вероятность в правой части неравенства убывает как экспонента от $\sqrt{n}$. Так что, $\mathbf{P}\left(\left|\xi_{n 1}\right|>\varepsilon\right) \leqslant e^{-\delta \sqrt{n}}$, и теорема 6.1 доказана.

(iv) Для вывода дополнения к теореме 6.1 заменим в предыдущих рассуждениях $t$ на последовательность $t_{n}, n=1,2, \ldots$, такую, что $t_{n} n / m \in[\alpha, \beta]$. Легко обнаружить, что все использованные в доказательстве теоремы 6.1 оценки могут быть выписаны в виде, не зависящем от $t$ при условии $t n / m \in[\alpha, \beta]$. Отсюда и вытекает результат.

6.2. Доказательство теорем 6.2, 6.3. (i) Для доказательства сходимости конечномерных распределений в теореме 6.2 сравним события под знаком вероятности

$$
\begin{aligned}
& \mathbf{P}\left(Y_{n}^{(t n)}\left(s_{i}\right) \in\left[a_{i}, b_{i}\right], i=1,2, \ldots, k, S_{n}>t n, M_{n-1} \leqslant t n\right) \\
& \mathbf{P}\left(Y_{n}^{(t n)}\left(s_{i}\right) \in\left[a_{i}, b_{i}\right], i=1,2, \ldots, k, S_{n}>t n\right)
\end{aligned}
$$

где $0<s_{1}<s_{2}<\cdots<s_{k}<1$. В (6.9) условие $S_{n}>t n$ не налагает ограничений на участки блуждания между точками $n s_{i-1}$ и $n s_{i}$ $\left(s_{0}=0\right), i \leqslant k$. В (6.8) требуется, чтобы блуждание на каждом участке не выходило за уровень $t n$, вероятность чего экспоненциально мала по $n$ с коэффициентом при $n$, б'ольшим чем соответствующий коэффициент при переходе блуждания из $s_{i-1} t n+d x_{i-1}$ в $s_{i} t n+d x_{i}$, и ею можно пренебречь. Таким образом, с точностью до пренебрежимо малой при $n \rightarrow \infty$ погрешности вероятность (6.8) переписывается в виде интеграла по области $\left[a_{1}, b_{1}\right] \times\left[a_{2}, b_{2}\right] \times \cdots \times\left[a_{k}, b_{k}\right]$ от

$$
\begin{gathered}
\mathbf{P}\left(Y_{n}^{(t n)}\left(s_{i}\right) \in d x_{i}, i \leqslant k\right) \mathbf{P}\left(S_{n\left(1-s_{k}\right)}>\operatorname{tn}\left(1-s_{k}\right)-x_{k} \sigma\left(\lambda_{t}\right) \sqrt{n}\right. \\
\left.M_{n\left(1-s_{k}\right)-1} \leqslant \operatorname{tn}\left(1-s_{k}\right)-x_{k} \sigma\left(\lambda_{t}\right) \sqrt{n}\right)
\end{gathered}
$$

а вероятность (6.9) переписывается в виде интеграла от

$$
\mathbf{P}\left(Y_{n}^{(t n)}\left(s_{i}\right) \in d x_{i}, i \leqslant k\right) \mathbf{P}\left(S_{n\left(1-s_{k}\right)}>\operatorname{tn}\left(1-s_{k}\right)-x_{k} \sigma\left(\lambda_{t}\right) \sqrt{n}\right),
$$

где мы считаем числа $n s_{i}$ целыми. Перейдем в (6.8), (6.9) к условным вероятностям и разделим второй множитель в $(6.10)$ на $\mathbf{P}(T(t n)=n)$, а в (6.11) - на $\mathbf{P}\left(S_{n}>t n\right)$. Вторые множители после применения асимптотик (3.3) и (1.4) перепишутся в следующем эквивалентном при $n \rightarrow \infty$ виде:

$$
\begin{aligned}
& c_{1}(t)\left(n\left(1-s_{k}\right)\right)^{-1 / 2} e^{-\psi\left(\theta_{n}\right) n\left(1-s_{k}\right)}\left[c_{1}(t) n^{-1 / 2} e^{-\psi(t) n}\right]^{-1}, \\
& a_{0}(t)\left(n\left(1-s_{k}\right)\right)^{-1 / 2} e^{-\psi\left(\theta_{n}\right) n\left(1-s_{k}\right)}\left[a_{0}(t) n^{-1 / 2} e^{-\psi(t) n}\right]^{-1},
\end{aligned}
$$

где для краткости мы положили $\theta_{n}:=t-x_{k} \sigma\left(\lambda_{t}\right) /\left(\left(1-s_{k}\right) \sqrt{n}\right)$. Таким образом, рассматриваемые условные вероятности эквивалентны, и остается воспользоваться теоремой 6.1 в части сходимости конечномерных распределений. 
(ii) Из теоремы 6.1 следует, что для любого $\varepsilon>0$ найдется компакт $\mathscr{K}_{\varepsilon} \subseteq C[0,1]$ такой, что $\mathbf{P}\left(Y_{n}^{(t n)}(\cdot) \in \overline{\mathscr{K}}_{\varepsilon} \mid S_{n}>t n\right)<\varepsilon$. В таком случае

$$
\begin{aligned}
\mathbf{P}\left(Y_{n}^{(t n)}(\cdot)\right. & \left.\in \overline{\mathscr{K}}_{\varepsilon} ; S_{n}>t n, M_{n-1} \leqslant t n\right) \leqslant \mathbf{P}\left(Y_{n}^{(t n)}(\cdot) \in \overline{\mathscr{K}}_{\varepsilon} ; S_{n}>t n\right) \\
& <\varepsilon \mathbf{P}\left(S_{n}>t n\right)=\varepsilon \frac{\mathbf{P}\left(S_{n}>t n\right)}{\mathbf{P}(T(t n)=n)} \mathbf{P}(T(t n)=n) \\
& \sim \varepsilon \frac{c_{0}(t)}{c_{1}(t)} \mathbf{P}(T(t n)=n)
\end{aligned}
$$

при $n \rightarrow \infty$. Переходя к условной вероятности, получаем, что семейство распределений вероятностей случайных элементов $\left(Y_{n}^{(t n)}(\cdot) \mid T(t n)=n\right)$ плотно. Теорема 6.2 доказана.

(iii) Для доказательства теоремы 6.3, во-первых, заметим, что все утверждения п. (i) выполняются равномерно по $t \in[\alpha, \beta]$. Во-вторых, для $t=t_{n} \in[\alpha, \beta]$ воспользуемся дополнением к теореме 6.1 и заметим, что существует компакт $\mathscr{K}_{\varepsilon}$ такой, что $\mathbf{P}\left(Y_{n}^{\left(t_{n} n\right)}(\cdot) \in \overline{\mathscr{K}}_{\varepsilon} \mid S_{n}>t_{n} n\right)<\varepsilon$, откуда, как и в п. (ii), следует плотность соответствующего семейства распределений.

(iv) Следствие 6.2 выводится буквальным перенесением доказательства теоремы 6.3 на условие $T(t n)=n, L_{1 n} \geqslant 0$.

6.3. Доказательство теоремы 6.5. Положив $t^{\prime}:=t n / k$ и заменив всюду в левой части (6.5) tn на $t^{\prime} k$, убеждаемся, что соотношение (6.5) достаточно установить при $k=n$. При $0 \leqslant a_{i}<b_{i}$, $i=0,1, \ldots, m$, преобразуем вероятность

$$
\mathbf{P}\left(S_{n}-t n \in\left(a_{0}, b_{0}\right], S_{n}-S_{n-i} \in\left[a_{i}, b_{i}\right], 1 \leqslant i \leqslant m, T(t n)=n\right)
$$

по схеме п. 3.1 и представим ее в виде произведения $e^{-\psi(t) n}$ на

$$
\int \cdots \int e^{-\lambda_{t}\left(s_{n}-t n\right)} \prod_{i=1}^{n} d F_{\lambda_{t}}\left(x_{i}\right)
$$

где интеграл берется по множеству $\left\{s_{n}-t n \in\left(a_{0}, b_{0}\right], s_{n}-s_{n-i} \in\right.$ $\left.\left[a_{i}, b_{i}\right], i \leqslant m, m_{n-1} \leqslant t n\right\}$. Перепишем $(6.13)$ в форме математического ожидания (ср. с (3.12)) и, переходя к обращенному случайному блужданию $\widehat{S}_{j}^{(t)}($ ср. с $(3.13))$, получим для него выражение

$$
\begin{gathered}
\mathbf{E}\left(e^{-\lambda_{t}\left(\widehat{S}_{n}^{(t)}-t n\right)} ; \widehat{S}_{i}^{(t)}-\widehat{S}_{n}^{(t)}+t n \in\left[a_{i}, b_{i}\right], 1 \leqslant i \leqslant m,\right. \\
\left.\widehat{S}_{n}^{(t)}-t n \in\left(a_{0}, b_{0}\right], \widehat{L}_{1 n}^{(t)} \geqslant \widehat{S}_{n}^{(t)}-t n\right) .
\end{gathered}
$$

Математическое ожидание (6.14) представим в интегральной форме:

$$
\int_{a_{0}}^{b_{0}} e^{-\lambda_{t} x} \mathbf{P}\left(\widehat{S}_{i}^{(t)}-x \in\left[a_{i}, b_{i}\right], 1 \leqslant i \leqslant m, \widehat{S}_{n}^{(t)}-t n \in d x, \widehat{L}_{1 n}^{(t)} \geqslant x\right) .
$$


Подобно п. 3.1, от интеграла (6.15) перейдем к суммам Дарбу-Стилтьеса и, воспользовавшись соотношением (2.4), получим, что верхняя и нижняя суммы эквивалентны при $n \rightarrow \infty$ выражению

$$
\begin{aligned}
& \left(\sqrt{2 \pi n} \sigma\left(\lambda_{t}\right)\right)^{-1} \int_{a_{0}}^{b_{0}} e^{-\lambda_{t} x} \int_{x+a_{m}}^{x+b_{m}} \mathbf{P}\left(L_{1}^{(t)} \geqslant x-y\right) \\
& \quad \times \mathbf{P}\left(S_{i}^{(t)} \in\left[x+a_{i}, x+b_{i}\right], i<m, S_{m}^{(t)} \in d y\right) d x .
\end{aligned}
$$

Домножим (6.16) на $e^{-\psi(t n)}$ и разделим на $\mathbf{P}(T(t n)=n)$. Применяя (3.3), получаем (6.5) при $m=n$. При этом

$$
\begin{aligned}
& \mathbf{P}\left(Y_{i}^{(t)} \in d y_{i}, 0 \leqslant i \leqslant m\right) \\
& \quad=\frac{1}{a(t)} e^{-\lambda_{t} y_{0}} \mathbf{P}\left(L_{1}^{(t)} \geqslant y_{0}-y_{m}\right) d y_{0} \mathbf{P}\left(S_{i}^{(t)} \in d y_{i}, 1 \leqslant i \leqslant m\right)
\end{aligned}
$$

в области $\left\{\left(y_{0}, y_{1}, \ldots, y_{m}\right): y_{0}>0, y_{i} \geqslant y_{0}, 1 \leqslant i \leqslant m\right\}$, в которой распределение вероятностей и сосредоточено. Как видно из (6.17), вектор $\left(Y_{1}^{(t)}, Y_{2}^{(t)}, \ldots, Y_{m}^{(t)} \mid Y_{0}^{(t)}\right)$ представляет собой отрезок однородной марковской цепи с переходной вероятностью при условии $Y_{0}=y_{0}$, равной

$$
\begin{aligned}
& \mathbf{P}\left(Y_{i+1}^{(t)} \in d y_{i+1} \mid Y_{i}^{(t)}=y_{i}\right) \\
& \quad=\frac{\mathbf{P}\left(L_{1}^{(t)} \geqslant y_{0}-y_{i+1}\right)}{\mathbf{P}\left(L_{1}^{(t)} \geqslant y_{0}-y_{i}\right)} \mathbf{P}\left(X^{(t)}+y_{i} \in d y_{i+1}\right),
\end{aligned}
$$

а вектор $\left(Y_{0}^{(t)}, Y_{1}^{(t)}, \ldots, Y_{m}^{(t)}\right)$ является смесью марковских цепей (6.18) по распределению начальной точки $Y_{0}^{(t)}$ :

$$
\mathbf{P}\left(Y_{0}^{(t)} \in d y_{0}\right)=\frac{1}{a(t)} e^{-\lambda_{t} y_{0}} \mathbf{P}\left(L_{1}^{(t)} \geqslant y_{0}\right) d y_{0} .
$$

7. Условные предельные теоремы для случайного блуждания и процесса ожидания при условии больших уклонений максимума.

Теорема 7.1 (см. [4]). При $\mu_{0} \geqslant 0$ для любого фиксированного $t \in$ $\left(\mu_{0}^{+}, \mu_{+}\right)$, а при $\mu_{0}<0$ для любого фиксированного $t \in\left(\gamma, \mu_{+}\right)$имеет место сходимость

$$
\left(Y_{n}^{(t n)}(\cdot) \mid M_{n}>t n\right) \stackrel{d}{\longrightarrow} w_{0}(\cdot) .
$$

3 а м е ч а н и е. В [4] соотношение (7.1) установлено в случае $\mu_{0} \geqslant$ 0 , но доказательство проходит и при $\mu_{0}<0$.

При $\mu_{0}<0$ и $t \in(0, \gamma]$ введем случайный элемент $\widehat{Y}_{n}^{(t n)}(\cdot) \in C[0,1]$, полагая $\hat{Y}_{n}^{(t n)}(s):=\left(S_{s T(t n)}-s t n\right) /\left(\sigma(\varkappa) \sqrt{t \gamma^{-1} n}\right)$ при $0 \leqslant s \leqslant 1$ таких, что значение $s T(t n)$ целое, и линейно интерполируя для остальных $s$. 
Теорема 7.2. При $\mu_{0}<0$ и любом фиксированном $t \in(0, \gamma]$

$$
\left(\widehat{Y}_{n}^{(t n)}(\cdot) \mid M_{n}>t n\right) \stackrel{d}{\longrightarrow} w_{0}(\cdot) .
$$

3 а м е ч а н и е. Ввиду теоремы 3.1(iii) соотношение (7.2) выполняется и при $t \in\left(\gamma, \mu_{+}\right)$.

Теорема 7.3. При $\mu_{0}<0$ справедливь следующие утверждения:

(i) для любого фиксированного $t \in\left(\gamma, \mu_{+}\right)$

$$
\mathbf{P}\left(Y_{n}^{(t n)}(\cdot) \mid M_{n}>t n, L_{1, n}>0\right) \stackrel{d}{\longrightarrow} w_{0}(\cdot) ;
$$

(ii) для любого фиксированного $t \in(0, \gamma]$

$$
\mathbf{P}\left(\widehat{Y}_{n}^{(t n)}(\cdot) \mid M_{n}>t n, L_{1, T(t n)}>0\right) \stackrel{d}{\longrightarrow} w_{0}(\cdot) .
$$

3 а м е ч а н и е. В соотношении (7.3) можно заменять независимо $Y_{n}^{(t n)}(\cdot)$ на $\widehat{Y}_{n}^{(t n)}(\cdot)$, а $L_{1, n}>0$ на $L_{1, T(t n)}>0$.

Положим

$$
Z_{m, k}^{(x)}(s):=\frac{W_{m+s(k-m)}-s x}{\sigma(\lambda(x /(k-m))) \sqrt{k-m}}
$$

при $0 \leqslant s \leqslant 1$ таких, что число $s(k-m)$ целое, и линейно интерполируем для остальных $0<s<1$. Напомним, что $\mathscr{T}(x)-$ первое пересечение процессом ожидания уровня $x>0$, а $\mathscr{T}_{0}(x)$ - ближайший слева к $\mathscr{T}(x)$ нуль процесса ожидания. Просматривая доказательства теорем 5.3-5.5 и применяя теорему 7.3 , приходим к следующему утверждению.

Теорема 7.4. При $\mu_{0}<0 u t \in\left(0, \mu_{+}\right)$имеет место следуюшая слабая сходимость в $C[0,1]:\left(Z_{\mathscr{T}_{0}(t n), \mathscr{T}(t n)}^{(t)}(\cdot) \mid \mathscr{M}_{n}^{W}>t n\right) \stackrel{d}{\longrightarrow} w_{0}(\cdot)$.

Следствие 7.1. При $t \in\left(0, \mu_{+}\right), t \neq \gamma$, траекторию прочесса ожидания $W_{k}, k \leqslant n$, можно разложить на три условно независимьх при условии $\mathscr{M}_{n}^{W}>$ tn участка: до $\mathscr{T}_{0}(t n)$, между $\mathscr{T}_{0}(t n)$ и $\mathscr{T}(t n)$, после $\mathscr{T}(t n)$. Последний участок - это свободныий прочесс ожидания, начинающийся в точке $W_{\mathscr{T}(t n)}$, случайной длительности $n-\mathscr{T}(t n)$. Первый участок - это прочесс ожидания, обрываюшийся в случайный момент $\mathscr{T}_{0}(t n)$, являющийся его нулем. При $t=\gamma$ первый и третий участки зависимь через их длительности.

7.1. Доказательство теорем 7.1, 7.2. (i) Пусть $H(\cdot)$ - произвольный непрерывный ограниченный функционал на $C[0,1]$. Покажем, что в условиях теоремы 7.1

$$
\mathbf{E} H\left(\left(Y_{n}^{(t n)}(\cdot) \mid M_{n}>t n\right)\right) \longrightarrow \mathbf{E} H\left(w_{0}(\cdot)\right) .
$$

Заметив, что

$$
\mathbf{E} H\left(\left(Y_{n}^{(t n)}(\cdot) \mid M_{n}>t n\right)\right)=\mathbf{E}\left(H\left(Y_{n}^{(t n)}(\cdot)\right) \mid M_{n}>t n\right),
$$


запишем для математического ожидания в (7.5) разложение

$$
\sum_{k=0}^{n-1} \frac{\mathbf{P}(T(t n)=n-k)}{\mathbf{P}\left(M_{n}>t n\right)} \mathbf{E}\left(H\left(Y_{n}^{(t n)}(\cdot)\right) \mid T(t n)=n-k\right) .
$$

Далее мы покажем, что для любого фиксированного $k$ и $t \in\left(\mu_{0}^{+}, \mu_{+}\right)$

$$
\mathbf{E}\left(H\left(Y_{n}^{(t n)}(\cdot)\right)-H\left(Y_{n-k}^{(t n)}(\cdot)\right) \mid T(t n)=n-k\right) \longrightarrow 0 .
$$

В силу $(6.3)\left(Y_{n-k}^{(t n)}(\cdot) \mid T(t n)=n-k\right) \stackrel{d}{\longrightarrow} w_{0}(\cdot)$. Поэтому из (7.8) следует, что математическое ожидание в (7.7) при любом фиксированном $k$ сходится при $n \rightarrow \infty$ к $\mathbf{E} H\left(w_{0}(\cdot)\right)$. Применяя теоремы 3.1(ii), 4.1 и 4.2, приходим к выводу, что при любом фиксированном $m$ сумма (7.7) по $k \leqslant m$ эквивалентна при $n \rightarrow \infty$ выражению

$$
\mathbf{E} H\left(w_{0}(\cdot)\right) \sum_{k=0}^{m} \theta^{-k}\left(\lambda_{t}\right)\left(1-\theta^{-1}\left(\lambda_{t}\right)\right)
$$

которое при $m \rightarrow \infty$ стремится к $\mathbf{E} H\left(w_{0}(\cdot)\right)$. Из доказательств теорем 4.1 и 4.2 следует, что $\sum_{k=m+1}^{n-1} \mathbf{P}(T(t n)=n-k)<\varepsilon \mathbf{P}\left(M_{n}>t n\right)$ для любого $\varepsilon>0$, достаточно большого $m$ и всех $n$. Тем самым, для доказательства теоремы 7.1 остается проверить утверждение (7.8).

Полагая для простоты число $m k / n$ целым, рассмотрим максимум по $m=1,2, \ldots, n$ модулей разностей

$$
Y_{n}^{(t n)}\left(\frac{m}{n}\right)-Y_{n-k}^{(t n)}\left(\frac{m}{n}\right)=\left(1+O\left(\frac{1}{n}\right)\right) \frac{S_{m}-S_{m-m k / n}}{\sigma\left(\lambda_{t}\right) \sqrt{n}}+S_{m} O\left(n^{-3 / 2}\right)
$$

при условии события $T(t n)=n-k$. В числителе дроби в правой части (7.10) стоит сумма $m k / n$ случайных величин $X_{i}$, которые мы рассматриваем при условии события $T(t n)=n-k$. Случайные величины $X_{i}$ с номерами $n-k+1, n-k+2, \ldots$ не зависят от этого события. Для с.в. $X_{i+1}, X_{i+2}, \ldots, X_{i+m k / n}$ при $i+m k / n \leqslant n-k$ оценим условную вероятность того, что максимум соответствующих величин (7.10) больше заданного $\delta>0$, суммой

$$
\sum_{i \leqslant n-k-m k / n} \mathbf{P}\left(\left|X_{i+1}+\cdots+X_{i+m k / n}\right|\left(\sigma\left(\lambda_{t}\right) \sqrt{n}\right)^{-1}>\delta \mid T(t n)=n-k\right) .
$$

Далее, имеем:

$$
\begin{aligned}
& \mathbf{P}\left(\left|X_{i+1}+\cdots+X_{i+m k / n}\right|\left(\sigma\left(\lambda_{t}\right) \sqrt{n}\right)^{-1}>\delta, T(t n)=n-k\right) \\
& \quad \leqslant k \mathbf{P}\left(\left|X_{i+1}\right|\left(\sigma\left(\lambda_{t}\right) \sqrt{n}\right)^{-1}>\frac{\delta}{k}, S_{n-k}>t n\right) .
\end{aligned}
$$

Применяя к вероятности в правой части (7.12) оценки п. 6.1(iii), приходим к заключению, что члены суммы (7.11) убывают экспоненциально 
по $\sqrt{n}$. То же верно и для самой суммы (7.11). Остается рассмотреть $k$ отрезков $X_{i+1}, X_{i+2}, \ldots, X_{i+m k / n}$, для которых $i+m k / n>n-k$. Для отрезка $X_{n-k+1}, X_{n-k+2}, \ldots, X_{n}$ имеем в силу неравенства Маркова:

$$
\begin{aligned}
& \mathbf{P}\left(\left|X_{n-k+1}+\cdots+X_{n}\right|\left(\sigma\left(\lambda_{t}\right) \sqrt{n}\right)^{-1}>\delta \mid T(t n)=n-k\right) \\
& \quad=\mathbf{P}\left(\left|X_{n-k+1}+\cdots+X_{n}\right|\left(\sigma\left(\lambda_{t}\right) \sqrt{n}\right)^{-1}>\delta\right) \leqslant k \frac{\mathbf{E}|X|}{\delta \sigma\left(\lambda_{t}\right) \sqrt{n}} .
\end{aligned}
$$

В промежуточном случае $n-k<i+m k / n<n$ воспользуемся оценками и типа (7.12), и типа (7.13). Остаточный член в правой части (7.10), очевидно, пренебрежимо мал, так что в итоге получаем:

$$
\mathbf{P}\left(\max _{1 \leqslant m \leqslant n}\left|Y_{n}^{(t n)}\left(\frac{m}{n}\right)-Y_{n-k}^{(t n)}\left(\frac{m}{n}\right)\right|>\delta \mid T(t n)=n-k\right) \longrightarrow 0 .
$$

Отсюда легко следует утверждение (7.8). Теорема 7.1 доказана.

(ii) Доказательство теоремы 7.2 проводится по схеме п. (i). Математическое ожидание

$$
\mathbf{E} H\left(\left(\widehat{Y}_{n}^{(t n)}(\cdot) \mid M_{n}>t n\right)\right) \equiv \mathbf{E}\left(H\left(\widehat{Y}_{n}^{(t n)}(\cdot)\right) \mid M_{n}>t n\right)
$$

разлагается в сумму

$$
\sum_{k=1}^{n} \frac{\mathbf{P}(T(t n)=k)}{\mathbf{P}\left(M_{n}>t n\right)} \mathbf{E}\left(H\left(\widehat{Y}_{n}^{(t n)}(\cdot)\right) \mid T(t n)=k\right) .
$$

Чтобы перейти в сумме (7.15) к пределу при $n \rightarrow \infty$, представим ее в виде интеграла. Положим $x_{k, n}:=\left(k-t \gamma^{-1} n\right) /\left(b^{-1}\left(t, t \gamma^{-1}\right) \sqrt{n}\right), 0 \leqslant k \leqslant n$, и определим на этом множестве точек вероятностную меру $Q_{n}$ :

$$
Q_{n}\left(\left\{x_{k, n}\right\}\right):=\mathbf{P}\left(T(t n)=k \mid M_{n}>t n\right) .
$$

Последовательность $Q_{n}$ слабо сходится к стандартному нормальному закону (см. (4.5)). Введем действительную функцию

$$
h_{n}(x):=\mathbf{E}\left(H\left(\widehat{Y}_{n}^{(t n)}(\cdot)\right) \mid T(t n)=k\right) \quad \text { при } x \in\left(x_{k-1, n}, x_{k, n}\right], 0 \leqslant k \leqslant n,
$$

доопределяя ее нулем при остальных $x$. Перепишем (7.15) в виде

$$
\int_{-\infty}^{\infty} h_{n}(x) Q_{n}(d x) .
$$

Для перехода к пределу в (7.17) воспользуемся следующим утверждением из книги П. Биллингсли (см. [10, теорема 5.5]).

Пусть $h_{n}(x), h(x)$ - измеримье действительнье функиии и $Q_{n}-$ последовательность вероятностных мер в $R$, слабо сходящаяся $\kappa Q$. 
Введем подмножество $D \subseteq \mathbf{R}$ чисел $x$, для которых существует последовательность $x_{n} \rightarrow x$ такая, что $h_{n}\left(x_{n}\right) \nrightarrow h(x)$ nри $n \rightarrow \infty$. Если $Q(D)=0$, то имеет место слабая сходимость

$$
Q_{n} h_{n}^{-1} \Rightarrow Q h^{-1}
$$

В нашем случае $Q-$ стандартная нормальная мера в $\mathbf{R}$, функция $h(x)$ постоянна и равна $\mathbf{E} H\left(w_{0}(\cdot)\right)$. Проверим, что множество $D$ пусто. Поскольку функция $h_{n}$ кусочно постоянна, последовательность $x_{n}$, сходящуюся к $x$, можно взять в виде $x_{n}=x_{k_{n}, n}$, где $k_{n}=t \gamma^{-1} n+O(\sqrt{n})$. Ввиду теоремы 6.3

$$
\mathbf{E}\left(H\left(Y_{k_{n}}^{(t n)}(\cdot)\right) \mid T(t n)=k_{n}\right) \longrightarrow \mathbf{E} H\left(w_{0}(\cdot)\right) .
$$

Покажем,что

$$
h_{n}\left(x_{k_{n}, n}\right) \equiv \mathbf{E}\left(H\left(\widehat{Y}_{n}^{(t n)}(\cdot)\right) \mid T(t n)=k_{n}\right) \longrightarrow \mathbf{E} H\left(w_{0}(\cdot)\right) .
$$

Если $s T(t n)$ целое, выразим $\widehat{Y}_{n}^{(t n)}(s)$ через $Y_{T(t n)}^{(t n)}(s)$ :

$$
\begin{aligned}
\widehat{Y}_{n}^{(t n)}(s) & =\frac{S_{s T(t n)}-s t n}{\sigma(\lambda(t n / T(t n)) \sqrt{T(t n)}} \frac{\sigma(\lambda(t n / T(t n)) \sqrt{T(t n)}}{\sigma(\varkappa) \sqrt{t \gamma^{-1} n}} \\
& \equiv Y_{T(t n)}^{(t n)}(s)\left(1+\delta_{n}(T(t n))\right), \quad \max \left(\delta_{n}(k): k-t \gamma^{-1} n=o(n)\right) \longrightarrow 0 .
\end{aligned}
$$

Отсюда следует, что последовательность $\left(\widehat{Y}_{n}^{(t n)}(\cdot) \mid T(t n)=k_{n}\right)$ имеет тот же предел по распределению, что и последовательность $\left(Y_{k_{n}}^{(t n)}(\cdot) \mid T(t n)=\right.$ $\left.k_{n}\right)$, т.е. выполняется (7.19).

Итак, для рассматриваемых $Q_{n}, h_{n}, Q, h$ верно (7.18). При этом $Q h^{-1}$ - вероятностная мера, целиком сосредоточенная в точке $\mathbf{E} H\left(w_{0}(\cdot)\right)$, а все меры $Q_{n} h_{n}^{-1}$ имеют носитель, содержащийся между минимумом $m$ и максимумом $M$ непрерывного ограниченного функционала $H$. Интеграл (7.17) перепишем в виде $\int_{m}^{M} y Q_{n} h_{n}^{-1}(d y)$. Переходя здесь к пределу при $n \rightarrow \infty$, получаем значение $\mathbf{E} H\left(w_{0}(\cdot)\right)$, которое и является пределом математического ожидания (7.14). Теорема 7.2 доказана.

7.2. Доказательство теоремы 7.3. Доказательство этой теоремы проводится по схеме п. 7.1. В случае (i) вместо разложения (7.7) берется его аналог

$$
\sum_{k=1}^{n-1} \frac{\mathbf{P}\left(T(t n)=n-k, L_{1 n}>0\right)}{\mathbf{P}\left(M_{n}>t n, L_{1 n}>0\right)} \mathbf{E}\left(H\left(Y_{n}^{(t n)}(\cdot)\right) \mid T(t n)=n-k, L_{1 n}>0\right) .
$$

В случае (ii) аналогично трансформируется разложение (7.15). 
8. Заключительные замечания. При $x \rightarrow \infty$ равномерно по $x / m \in[\alpha, \beta] \subseteq\left(\mu_{0}, \mu_{+}\right)$

$$
\mathbf{P}(T(x)=m) \sim c_{1}\left(\frac{x}{m}\right) m^{-1 / 2} \exp \left\{-\left(\psi\left(\frac{x}{m}\right) \frac{m}{x}\right) x\right\}
$$

(см. (3.6)), а при $\mu_{0}<0$ равномерно по $k$ таким, что $k-\gamma^{-1} x=o\left(x^{2 / 3}\right)$,

$$
\mathbf{P}(T(x)=k) \sim \frac{1}{\sigma(\varkappa) \gamma^{-3 / 2} \sqrt{x}} \varphi\left(\frac{k-\gamma^{-1} x}{\sigma(\varkappa) \gamma^{-3 / 2} \sqrt{x}}\right) \gamma^{-1} a(\gamma) e^{-\varkappa x}, \quad x \rightarrow \infty
$$

(см. (3.8)). При $\mu_{0}<0$ функция $\psi(x / y) y / x$ по переменной $y$ достигает минимума в точке $y=x \gamma^{-1}$, строго возрастая в стороны от этой точки. Применяя технику п. 4 , получаем известную оценку КрамераЛундберга [12, п. 1.2]: при $\mu_{0}<0$

$$
\mathbf{P}(M>x) \sim \gamma^{-1} a(\gamma) e^{-\varkappa x}, \quad x \rightarrow \infty .
$$

Из (8.2), (8.3) находим, что равномерно по $k$ таким, что $k-\gamma^{-1} x=$ $o\left(x^{2 / 3}\right)$,

$$
\mathbf{P}(T(x)=k \mid M>x) \sim \frac{1}{\sigma(\varkappa) \gamma^{-3 / 2} \sqrt{x}} \varphi\left(\frac{k-\gamma^{-1} x}{\sigma(\varkappa) \gamma^{-3 / 2} \sqrt{x}}\right), \quad x \rightarrow \infty,
$$

т.е. для с.в. $\left(T(x)-\gamma^{-1} x\right) /\left(\sigma(\varkappa) \gamma^{-3 / 2} \sqrt{x}\right)$ имеет место локальная предельная теорема при условии $T(x)<\infty, x \rightarrow \infty$.

Пересечение случайным блужданием высокого уровня $x$ (событие $\{M>x\})$ реализуется на траекториях $\left(m, S_{m}\right), m=0,1,2, \ldots$, которые на участке $m \in[0, T(x)]$ располагаются на координатной плоскости в окрестности прямой, исходящей из начала координат, с тангенсом угла наклона $\gamma$. Если блуждание ограничено отрезком $[0, n]$, где $n \geqslant \gamma^{-1} x$, то пересечение уровня $x$ происходит на траекториях, которые до момента $T(x)$ те же, что при реализации события $\{M>x\}$. Если же $n<\gamma^{-1} x$, то соответствующие траектории случайного блуждания близки к прямой, идущей из начала координат в точку $(n, x)$.

После передачи рукописи в редакцию автор познакомился с работой С. Асмуссена [13], в которой установлена интегральная теорема об асимптотической нормальности с.в. $\left(T(x)-\gamma^{-1} x\right) /\left(\sigma(\varkappa) \gamma^{-3 / 2} \sqrt{x}\right)$ при условии $T(x)<\infty, x \rightarrow \infty$. Там также доказана сходимость к броуновскому мосту процесса

$$
\left(\frac{S_{[s T(x)]}-s x}{\sigma(\varkappa) \gamma^{-3 / 2} \sqrt{x}} \mid T(x)<\infty\right), \quad 0 \leqslant s \leqslant 1 .
$$

Этот результат нетрудно получить из соотношений (6.3), (8.1), (8.2), применяя схему рассуждений раздела 4. 
Автор выражает искреннюю признательность анонимному рецензенту, по совету которого компоновка работы была полностью пересмотрена и были внесены правки технического характера. Автору приятно поблагодарить Д. А. Коршунова, прочитавшего рукопись и сделавшего полезные замечания.

\section{СПИСОК ЛИТЕРАТУРЫ}

1. Боровков А. А., Кориунов Д. А. Вероятности больших уклонений одномерных цепей Маркова. Часть 2. Достационарные распределения в экспоненциальном случае. - Теория вероятн. и ее примен., 2000, т. 45, в. 3, с. 437-468.

2. Боровков A. А. Эргодичность и устойчивость случайных процессов. М.: Эдиториал УРСС, 1999, $440 \mathrm{c.}$

3. Феллер В. Введение в теорию вероятностей и ее приложения, т. 2. М.: Мир, 1984, $751 \mathrm{c.}$

4. Шкляев $A . B$. Предельные теоремы для случайного блуждания при условии большого уклонения максимума. - Теория вероятн. и ее примен., 2010, т. 55, в. 3, c. $590-598$.

5. Петров В. В. О вероятностях больших уклонений сумм независимых случайных величин. - Теория вероятн. и ее примен., 1965, т. 10, в. 2, с. 310-322.

6. Козлов М.В. О больших уклонениях строго докритических ветвящихся процессов в случайной среде с геометрическим распределением числа потомков. - Теория вероятн. и ее примен., 2009, т. 54, в. 3, с. 439-465.

7. Боровков A.A. О преобразовании Крамера, больших уклонениях в граничных задачах и условном принципе инвариантности. - Сиб. матем. журн., 1995, т. 36, № 3 , с. 493-509.

8. Боровков A.A. Об условных распределениях, связанных с большими уклонениями. - Сиб. матем. журн., 1996, т. 37, № 4, с. 732-744.

9. Полещук О. М. Большие уклонения и условные предельные теоремы. Дис. ... канд. физ.-матем. наук. М.: МГУ, 1989.

10. Биллингсли П. Сходимость вероятностных мер. М.: Наука, 1977, 352 с.

11. Шкляев A.В. Большие уклонения и предельные теоремы для некоторых функционалов от случайного блуждания. Дис. ... канд. физ.-матем. наук. М.: МГУ, 2011.

12. Embrechts P., Klüppelberg C., Mikosch T. Modelling Extremal Events. Berlin: Springer-Verlag, 1997, 645 p. (Appl. Math. (N.Y.), v. 33.)

13. Asmussen $S$. Conditioned limit theorems relating a random walk to its associate, with applications to risk reserve process and the GI/G/1 queue. - Adv. Appl. Probab., 1982, v. 14, № 1, p. 143-170.

Поступила в редакцию

15.XII. 2011

Исправленный вариант

15.V.2012 